\title{
Real-time Active and Reactive Power Regulation in Power Systems with Tap-changing Transformers and Controllable Loads *
}

\author{
Xuan Zhang, Ren Kang, Malcolm McCulloch and Antonis Papachristodoulou ${ }^{\mathrm{a}, 1}$ \\ ${ }^{a}$ Department of Engineering Science, University of Oxford, Parks Road, Oxford, OX1 3PJ, United Kingdom
}

\begin{abstract}
Due to increasing uncertainties resulting from renewable energy penetration and variability in both supply and demand, the control and economic optimization for power networks will need to run on faster time-scales. Moreover, distributed and decentralized control architectures are necessary as power systems are distributed large-scale networks with a lot of complexity, which makes centralized control expensive, inefficient and hard to implement. In this paper, the problem of real-time active and reactive power regulation for power networks with controllable loads and tap-changing transformers is considered. First, the state-space model of a transmission level network under exogenous disturbances is obtained, which is decomposed into two subsystems, describing frequency and voltage dynamics respectively. For the subsystem describing frequency dynamics, an optimization problem relating to active power regulation is first formulated. A distributed controller is then proposed and its optimality, stability and delay robustness are studied. Also, nonlinear proportional actions for controllable loads are introduced to improve the performance of the subsystem. For the subsystem describing voltage dynamics, simple integral control is used for controllable loads and the asymptotic stability of the closed-loop subsystem is shown. To implement the reactive power regulation scheme, a method for local users to detect the tap position variation of the On-Load Tap Changer (OLTC) at the bus they are connected to is presented. Thus, the overall load control scheme is completely decentralized and can be applied easily. Numerical investigations illustrate the performance of the combined active and reactive power regulation scheme.
\end{abstract}

Key words: Power system, optimization, distributed control, controllable load, tap-changing transformer.

\section{Introduction}

The operation and control of the modern power grid is layered. Each layer is characterized by its operational/control objectives, corresponding to one time-

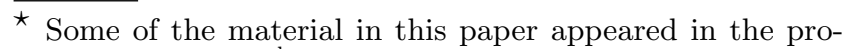
ceedings of the $53^{\text {rd }}$ IEEE Conference on Decision and Control, December 15-17, 2014, Los Angeles, CA, USA. The work of X. Zhang was supported by the China Scholarship Council (CSC). The work of A. Papachristodoulou was supported in part by the Engineering and Physical Sciences Research Council projects EP/J012041/1, EP/J010537/1 and EP/M002454/1. R. Kang and M. McCulloch would like to thank Martin Lee from Southern and Scottish Energy Power Distribution for providing the tap change data and advice on the detection algorithms.

Email address: \{xuan.zhang, ren.kang, malcolm.mcculloch, antonis\} @eng.ox.ac.uk (Xuan Zhang, Ren Kang, Malcolm McCulloch and Antonis Papachristodoulou).

1 Tel.:+44 18652 83036; Fax: +44 1865273010 .
}

scale. When looking at one of the layers, the influence from other layers can be ignored because of this architecture. For example, if considering long term contracts (slow time-scale) between a generation company and a factory, the operation and control in the transmission network (fast time-scale) is neglected. This separation works well when the time-scales of the two layers are significantly different. Even if the difference is less obvious, e.g., between seconds and minutes, it is still acceptable because of high predictability and low uncertainty in the system [1]. However, as renewable energy, distributed generation and dynamic demand are introduced, the power grid will face increasing uncertainty and variability in both supply and demand, especially in the real-time layer (time-scale of usually 5 minutes). In order to maintain system-wide efficiency, reliability and robustness under these changes, redesigning conventional controls and actions and merging different layers/time-scales together becomes more and more necessary. On the other hand, power systems are large-scale and complicated. Because of the increase in uncertainty and variability, conventional centralized 
control for power networks becomes expensive and less efficient. Therefore, distributed and decentralized control frameworks are necessary, not only to deal with the problem of scale, but also to reduce the requirement for communication which can affect system control and optimization in real-time.

The redesign of conventional control approaches and actions that combines different layers/time-scales together, in either distributed, decentralized or centralized manners is an area of active research. For example, [1] presented a novel control scheme for achieving optimal power balancing and congestion management in power systems via nodal prices, and introduced the concept of autonomous power networks. In [2,3], a distributed realtime control architecture was proposed which merged primary, secondary, and tertiary control for power networks, so as to achieve system-wide efficiency and reliability. In [4], the authors proposed both a decentralized controller and a centralized controller for frequency regulation and generation cost minimization in power systems. In [5], conventional Automatic Generation Control (AGC) was combined with Economic Dispatch (ED) automatically and dynamically, providing an optimization viewpoint to modify AGC. In [6], the authors designed a distributed real-time frequency control scheme through reverse- and forward-engineering. In [7], a hierarchical transactive control architecture was presented which combined market transactions at three different time-scales. In [8], decentralized and distributed primary, secondary, and tertiary control strategies in microgrids were studied, showing that the adoption of droop control was necessary and sufficient to achieve economic optimization in microgrids. In [9], a fast-acting decentralized load control scheme was presented for primary frequency regulation in power networks. In [10], a frequency preserving optimal load control scheme was proposed, which was used to balance power and restore the nominal frequency after a disturbance.

In this paper, conventional primary and secondary control for power systems with tap-changing transformers [11] are merged and redesigned. Moreover, the participation of controllable loads [12] in both active and reactive power regulation is considered, which is unlike most previous work. As shown in [12,13], the use of controllable loads can provide significant added stability and robustness to power systems, especially under real-time disturbances, contingency and renewable energy penetration. The contribution of this paper includes: (i) a distributed generation control scheme which asymptotically stabilizes the frequency dynamics regardless of communication delay and minimizes aggregate power generation cost; (ii) a decentralized load control scheme which consists of proportional actions in real power regulation and integral actions in reactive power regulation, and is simple for realization; and (iii) a method for determining tap change events which can be used to estimate the tap position variation of On-
Load Tap Changers (OLTCs), thus, assisting decision making for the load control scheme.

The rest of the paper is organized as follows: In Section 2, the complete state-space model of a transmission level network under exogenous disturbances is presented, where the system is decomposed into two subsystems, i.e., frequency dynamics and voltage dynamics. In Section 3, a distributed generation control scheme and a decentralized load control scheme are proposed to regulate active power for the subsystem describing frequency dynamics, and the optimality, stability and delay robustness of the closed-loop subsystem are shown. In Section 4, a decentralized reactive power regulation scheme for the subsystem describing voltage dynamics is proposed. In order to implement this (reactive power control) scheme, a method for detecting tap position variation of OLTCs at the end users is proposed. In Section 5, numerical examples are presented to illustrate the properties of the control scheme. Conclusions and future work are presented in Section 6 .

\subsection{Notation}

$|\mathcal{X}|$ is the cardinality of a set $\mathcal{X} . x^{0}$ denotes the nominal value of a state variable $x . x^{T}$ denotes the transpose of $x \cdot \operatorname{diag}\{\star\}$ denotes a diagonal matrix with corresponding entries $\star$ on the main diagonal. $X \in \mathbb{R}^{m \times n}$ or $X=[\star]_{m \times n}$ is an $m \times n$ real matrix. $\mathbf{1}_{m \times n}\left(\mathbf{0}_{m \times n}\right)$ denotes an $m$ by $n$ matrix whose entries are $1(0)$ and $I_{m}$ denotes an identity matrix of size $m \times m . x^{*}$ denotes the equilibrium of a state variable $x \cdot \operatorname{tr}(X)$ is the trace of a square matrix $X . X \succeq 0(X \succ 0)$ denotes that a square matrix $X$ is positive semi-definite (positive definite). $\mathcal{C}_{n, \tau}$ denotes the Banach space of continuous functions mapping the interval $[-\tau, 0]$ into $\mathbb{R}^{n}$ with the topology of uniform convergence, where $\mathbb{R}^{n}$ is the $n$-dimensional Euclidean space. The norm on $\mathcal{C}_{n, \tau}$ is defined as $\|\phi\|_{\mathcal{C}}=\sup _{-\tau<\theta<0}|\phi(\theta)|$ where $|\star|$ is the Euclidean norm for vectors and the induced matrix norm for matrices. Finally, the projection of a function $h(y)$ on a variable $x \in[\underline{x}, \bar{x}]$ is denoted by $(h(y))_{\underline{x}}^{\bar{x}}$ where

$$
(h(y))_{\underline{x}}^{\bar{x}}= \begin{cases}\min \{0, h(y)\} & \text { if } x=\bar{x} \\ h(y) & \text { if } \underline{x}<x<\bar{x} . \\ \max \{0, h(y)\} & \text { if } x=\underline{x}\end{cases}
$$

\section{System Model}

\subsection{Network Model}

Consider a transmission (subtransmission) level network with arbitrary topology, described by a connected directed graph $(\mathcal{G} \cup \mathcal{L}, \mathcal{E})$. Here $\mathcal{G}$ is the set of generator buses, $\mathcal{L}$ is the set of load buses, and $\mathcal{E} \subseteq(\mathcal{G} \cup \mathcal{L}) \times$ $(\mathcal{G} \cup \mathcal{L})$ is the set of transmission lines connecting the 
buses. Each generator bus contains only one synchronous generator/generation unit, and each load bus contains only one load which is an aggregation of a certain amount of users at the bus it is connected to (alternatively speaking, each load corresponds to a distribution level network). This can be realized by introducing fictitious buses [14]. Moreover, all load buses are equipped with transformers containing OLTCs. Number the generator buses $1, \cdots, m(\mathcal{G}=\{1, \cdots, m\})$, the load buses $m+1, \cdots, m+n(\mathcal{L}=\{m+1, \cdots, m+n\})$, and the transmission lines $1, \cdots, p$ corresponding to a lexicographic ordering where $p=|\mathcal{E}|$. Define an orientation from bus $i$ to bus $j$ if $(i, j) \in \mathcal{E}, i<j$. View all buses as voltage sources. Denote the voltage of each generator bus by $v_{i} \angle \delta_{i}, i \in \mathcal{G}$, and the primary-side voltage of the OLTC at each load bus by $v_{i} \angle \delta_{i}, i \in \mathcal{L}$. Assume the network is working around a nominal operating point which is determined by an ED problem at a more slower timescale. To simplify the notation, in the rest of the paper, all state variables denote time-varying deviations from their nominal operating values, and moreover, all state variables with tilde denote their nominal operating values plus their time-varying deviations (from the nominal operating values), i.e., $\tilde{x}=x^{0}+x$ where $x$ is the timevarying deviation $\left(x^{*}\right.$ denotes the equilibrium of $x$ ).

Generator bus model. First, the following assumption holds for the generator bus model.

Assumption 1 Generator bus voltage magnitudes are fixed, i.e., $v_{i}=0, \forall i \in \mathcal{G}$ (alternatively, $\tilde{v}_{i}=v_{i}^{0}$ ).

This is reasonable since generator bus voltage magnitudes can be well and fast controlled through excitation systems/Automatic Voltage Regulators (AVRs) [15] when the system is operating around a nominal state. Similar assumptions are also used in [16-18]. Let $\omega_{i}=\dot{\delta}_{i}$ be the time-varying frequency deviation from the nominal frequency $\omega_{i}^{0}$, e.g., $2 \pi \times 50 \mathrm{~Hz}$ in Europe, at bus $i, i \in \mathcal{G} \bigcup \mathcal{L}$. The dynamics of generator buses are given by the (linearized) swing equations [14]

$$
M_{i} \dot{\omega}_{i}+D_{i} \omega_{i}=P_{M_{i}}-P_{i}-\sum_{(i, j) \in \mathcal{E}} P_{i j}, i \in \mathcal{G}
$$

where $M_{i}>0$ is the generator inertia, $D_{i}>0$ is the damping coefficient, $P_{M_{i}}$ is the mechanical power input, $P_{i}$ is the disturbance injection, e.g., renewable energy injections and variations on the supply side, and $P_{i j}$ is the active power transmitted from bus $i$ to bus $j$ if they are connected. Moreover, in realistic power systems, each generator contains a turbine-governor system $[15,5]$ :

$$
\dot{P}_{M_{i}}=\frac{1}{T_{T G_{i}}}\left(P_{C_{i}}-P_{M_{i}}-\frac{\omega_{i}}{R_{i}}\right), i \in \mathcal{G}
$$

where $P_{C_{i}}$ is the command/control input to the generator and $T_{T G_{i}}, R_{i}>0$ are constant parameters. Note that the dynamics of the turbine-governor system have been simplified using a first-order model as in [5].

Remark 1 By identifying groups of generators that are closely coupled internally but weakly coupled between groups, the transmission network can be divided into control areas/subsystems. Each control area contains a tie-line bias control through which active power regulation is allocated to generators in this area via participation factors. The dynamics of the tie-line bias control are given by [15]

$$
\dot{P}_{C_{i}}=K_{i}\left(-B_{i} \omega_{i}-P_{\text {tie-line }, i}\right)
$$

where $P_{C_{i}}$ is the total command input to generators in area $i, \omega_{i}$ is the frequency deviation of area $i$ (the dynamics of $\omega_{i}$ are obtained through aggregation techniques [19,15]), $P_{\text {tie-line, } i}$ is the total tie-line active power transmitted from area $i$ to neighbourhood areas, and $K_{i}, B_{i}>0$ are constant (design) parameters. In this paper, we do not specify control areas and do not adopt the conventional tie-line bias control, however, the control input $P_{C_{i}}$ in (2) will be designed to be as similar to (3) as possible in order to achieve more economic efficiency. Thus, the controller developed in this paper can also be used as a redesigned generation control scheme/tie-line bias control at the control area level.

Transmission system model. The standard decoupling approximation in power flows $[20,21]$ is used here.

Assumption 2 The resistance of transmission lines is negligible and $\sin \left(\tilde{\delta}_{i}-\tilde{\delta}_{j}\right) \approx \tilde{\delta}_{i}-\tilde{\delta}_{j}, \cos \left(\tilde{\delta}_{i}-\tilde{\delta}_{j}\right) \approx 1$ hold.

This assumption is reasonable as: (i) realistic transmission networks are very close to being lossless [21,15]; (ii) the physical coupling between active power flows and bus voltage magnitudes, and between reactive power flows and bus angle differences is weak [20,21]. Similar assumptions are also used in $[17,22]$. Under Assumptions $1-2$, active and reactive power flows in transmission lines are described by [23]

$$
\begin{aligned}
\tilde{P}_{i j} & =\tilde{v}_{i} \tilde{v}_{j} B_{i j} \sin \left(\tilde{\delta}_{i}-\tilde{\delta}_{j}\right)=\tilde{v}_{i} \tilde{v}_{j} B_{i j}\left(\tilde{\delta}_{i}-\tilde{\delta}_{j}\right) \\
\tilde{Q}_{i j} & =\tilde{v}_{i}^{2} B_{i j}-\tilde{v}_{i} \tilde{v}_{j} B_{i j} \cos \left(\tilde{\delta}_{i}-\tilde{\delta}_{j}\right)=\tilde{v}_{i}^{2} B_{i j}-\tilde{v}_{i} \tilde{v}_{j} B_{i j}
\end{aligned}
$$

where $(i, j) \in \mathcal{E}$ and $B_{i j}=B_{j i}>0$ is the susceptance of the line connecting buses $i$ and $j$. This leads to

$$
\begin{array}{cr}
P_{i j}= & v_{i}^{0} v_{j}^{0} B_{i j}\left(\delta_{i}-\delta_{j}\right), i, j \in \mathcal{G} \\
P_{i j}= & v_{i}^{0} B_{i j}\left(\delta_{i}^{0}-\delta_{j}^{0}\right) v_{j}+v_{i}^{0} v_{j}^{0} B_{i j}\left(\delta_{i}-\delta_{j}\right), i \in \mathcal{G}, j \in \underset{\mathcal{L}}{(4 \mathrm{a})} \\
P_{i j}= & v_{j}^{0} B_{i j}\left(\delta_{i}^{0}-\delta_{j}^{0}\right) v_{i}+v_{i}^{0} v_{j}^{0} B_{i j}\left(\delta_{i}-\delta_{j}\right), i \in \mathcal{L}, j \in \mathcal{G} \\
P_{i j}= & v_{j}^{0} B_{i j}\left(\delta_{i}^{0}-\delta_{j}^{0}\right) v_{i}+v_{i}^{0} B_{i j}\left(\delta_{i}^{0}-\delta_{j}^{0}\right) v_{j} \\
& +v_{i}^{0} v_{j}^{0} B_{i j}\left(\delta_{i}-\delta_{j}\right), i, j \in \mathcal{L}
\end{array}
$$




$$
\begin{aligned}
Q_{i j} & =0, i, j \in \mathcal{G} \\
Q_{i j} & =-v_{i}^{0} B_{i j} v_{j}, i \in \mathcal{G}, j \in \mathcal{L} \\
Q_{i j} & =\left(2 v_{i}^{0}-v_{j}^{0}\right) B_{i j} v_{i}, i \in \mathcal{L}, j \in \mathcal{G} \\
Q_{i j} & =\left(2 v_{i}^{0}-v_{j}^{0}\right) B_{i j} v_{i}-v_{i}^{0} B_{i j} v_{j}, i, j \in \mathcal{L} .
\end{aligned}
$$

Load bus model. By considering both angle dynamics and voltage dynamics at load buses, the model is described as $[16,23,24]$

$$
\begin{gathered}
\tilde{P}_{V F_{i}}\left(\tilde{n}_{i} \tilde{v}_{i}, \omega_{i}, \tilde{p}_{i}\right)+\tilde{P}_{i}=-\sum_{(i, j) \in \mathcal{E}} \tilde{P}_{i j}, i \in \mathcal{L} \\
\tilde{Q}_{V_{i}}\left(\tilde{n}_{i} \tilde{v}_{i}, \tilde{q}_{i}\right)+\tilde{Q}_{i}=-\epsilon_{i} \tilde{v}_{i} \dot{\tilde{v}}_{i}-\tilde{Q}_{S_{i}}-\sum_{(i, j) \in \mathcal{E}} \tilde{Q}_{i j}, i \in \mathcal{L} \\
\dot{\tilde{n}}_{i}=\frac{1}{T_{T_{i}}}\left(\tilde{v}_{\mathrm{ref}, i}-\tilde{n}_{i} \tilde{v}_{i}\right), i \in \mathcal{L} .
\end{gathered}
$$

In the above equations, $\tilde{P}_{V F_{i}}\left(\tilde{n}_{i} \tilde{v}_{i}, \omega_{i}, \tilde{p}_{i}\right)$ is the real load demand, $\tilde{n}_{i} \tilde{v}_{i}$ is the secondary-side voltage magnitude of the OLTC at load bus $i$ where $1: \tilde{n}_{i}$ is the tap ratio of the OLTC, $\tilde{p}_{i}$ is the control variable of the controllable part in real power consumption, $\tilde{Q}_{V_{i}}\left(\tilde{n}_{i} \tilde{v}_{i}, \tilde{q}_{i}\right)$ is the reactive load demand, $\tilde{q}_{i}$ is the control variable of the controllable part in reactive power consumption. Also, $\tilde{P}_{i}$ and $\tilde{Q}_{i}$ are the active part and reactive part of disturbance injection respectively, e.g., variations on the demand side and renewable energy injections, and $P_{i}^{0}=0$ and $Q_{i}^{0}=0$ hold at the nominal state. The term $\epsilon_{i} \tilde{v}_{i} \dot{\tilde{v}}_{i}$ in Equation (5b) is the result of singular perturbation $[23,25,24]$ where $\epsilon_{i}$ is a small positive parameter [25], and $\tilde{Q}_{S_{i}}=\tilde{v}_{i}^{2} B_{\operatorname{sh}, i}$ is the reactive power injection due to shunt reactors where $B_{\mathrm{sh}, i}>0$ is the shunt susceptance (in practical transmission networks, shunt reactors are usually employed at the end of lines/transformers to compensate for the effect of line capacitance) $[20,26,27]$. The dynamics of OLTCs are given by $(5 \mathrm{c})[17,11]$, where $T_{T_{i}}>0$ is the time constant of the tap changer, and $\tilde{v}_{\text {ref, } i}$ is the secondary-side reference setting of the OLTC which is a given constant to maintain certain voltage levels for users in the distribution network.

Remark 2 Real load demand at each load bus may depend on the voltage magnitude $\tilde{n}_{i} \tilde{v}_{i}$, or the frequency $\omega_{i}^{0}+\omega_{i}$ (since $\omega_{i}^{0}$ is constant for all buses, the dependence is regardless of $\left.\omega_{i}^{0}\right)$, or both of them. The controllable part of real load demand also depends on the control input $\tilde{p}_{i}$. So $\tilde{P}_{V F_{i}}\left(\tilde{n}_{i} \tilde{v}_{i}, \omega_{i}, \tilde{p}_{i}\right)$ is used in (5a). Note that constant real load consumption can be included in $\tilde{P}_{V F_{i}}\left(\tilde{n}_{i} \tilde{v}_{i}, \omega_{i}, \tilde{p}_{i}\right)$. Reactive load demand only depends on the voltage magnitude $\tilde{n}_{i} \tilde{v}_{i}$ as described in [16,23,24]. The controllable part of reactive load demand depends on the control input $\tilde{q}_{i}$ as well. So $\tilde{Q}_{V_{i}}\left(\tilde{n}_{i} \tilde{v}_{i}, \tilde{q}_{i}\right)$ is used in (5b), which can also include constant reactive load consumption. Moreover, active voltage control devices such as capacitor banks and synchronous condensers [20,18] in distribution networks are included in each load.

Linearize (5) around the nominal operating point to get

$$
D_{i} \omega_{i}+F_{i}\left(n_{i}^{0} v_{i}+v_{i}^{0} n_{i}\right)+P_{L_{i}}+P_{i}=-\sum_{(i, j) \in \mathcal{E}} P_{i j}, i \in \mathcal{L}
$$

$$
\begin{array}{r}
\epsilon_{i} v_{i}^{0} \dot{v}_{i}+E_{i}\left(n_{i}^{0} v_{i}+v_{i}^{0} n_{i}\right)+Q_{L_{i}}+Q_{i}+Q_{S_{i}}=-\sum_{(i, j) \in \mathcal{E}} Q_{i j} \\
\dot{n}_{i}=-\frac{1}{T_{T_{i}}}\left(n_{i}^{0} v_{i}+v_{i}^{0} n_{i}\right), \quad i \in \mathcal{L} \quad(6 \mathrm{~b}) \\
\quad(6 \mathrm{c})
\end{array}
$$

where

$$
\begin{gathered}
D_{i}=\left.\frac{\partial \tilde{P}_{V F_{i}}\left(\tilde{n}_{i} \tilde{v}_{i}, \omega_{i}, \tilde{p}_{i}\right)}{\partial \omega_{i}}\right|_{\omega_{i}=0, \tilde{n}_{i} \tilde{v}_{i}=n_{i}^{0} v_{i}^{0}, \tilde{p}_{i}=p_{i}^{0}} \\
F_{i}=\left.\frac{\partial \tilde{P}_{V F_{i}}\left(\tilde{n}_{i} \tilde{v}_{i}, \omega_{i}, \tilde{p}_{i}\right)}{\partial\left(\tilde{n}_{i} \tilde{v}_{i}\right)}\right|_{\omega_{i}=0, \tilde{n}_{i} \tilde{v}_{i}=n_{i}^{0} v_{i}^{0}, \tilde{p}_{i}=p_{i}^{0}} \\
P_{L_{i}}=\left.p_{i} \frac{\partial \tilde{P}_{V F_{i}}\left(\tilde{n}_{i} \tilde{v}_{i}, \omega_{i}, \tilde{p}_{i}\right)}{\partial \tilde{p}_{i}}\right|_{\omega_{i}=0, \tilde{n}_{i} \tilde{v}_{i}=n_{i}^{0} v_{i}^{0}, \tilde{p}_{i}=p_{i}^{0}} \\
E_{i}=\left.\frac{\partial \tilde{Q}_{V_{i}}\left(\tilde{n}_{i} \tilde{v}_{i}, \tilde{q}_{i}\right)}{\partial\left(\tilde{n}_{i} \tilde{v}_{i}\right)}\right|_{\tilde{n}_{i} \tilde{v}_{i}=n_{i}^{0} v_{i}^{0}, \tilde{q}_{i}=q_{i}^{0}} \\
Q_{L_{i}}=\left.q_{i} \frac{\partial \tilde{Q}_{V_{i}}\left(\tilde{n}_{i} \tilde{v}_{i}, \tilde{q}_{i}\right)}{\partial \tilde{q}_{i}}\right|_{\tilde{n}_{i} \tilde{v}_{i}=n_{i}^{0} v_{i}^{0}, \tilde{q}_{i}=q_{i}^{0}} \\
Q_{S_{i}}=2 v_{i}^{0} B_{\mathrm{sh}, i} v_{i} .
\end{gathered}
$$

According to the natural static characteristics of loads and active voltage control devices (within the control range) $[20,18,28], D_{i}>0, F_{i}>0, E_{i}>0$ hold at the nominal operating point. Moreover, $n_{i}^{0}>0, v_{i}^{0}>0$ hold.

\subsection{State-space Description}

In this subsection, the state-space description of the dynamics given by (1)-(2), (4), and (6) is presented. Under Assumption 2, this system naturally decomposes into two subsystems, i.e., Subsystem I (1)-(2), (4a)-(4d) and (6a), and Subsystem II (4e)-(4h) and (6b)-(6c). The outputs of Subsystem II $v_{i}$ and $n_{i}^{0} v_{i}+v_{i}^{0} n_{i}$ are the inputs to Subsystem I.

Subsystem I. Let $\delta_{i j}=\delta_{i}-\delta_{j},(i, j) \in \mathcal{E}$. Subsystem I can be rearranged as

$$
\begin{gathered}
M_{i} \dot{\omega}_{i}+D_{i} \omega_{i}=P_{M_{i}}-P_{i}^{\mathcal{G}}-\sum_{(i, j) \in \mathcal{E}} T_{i j} \delta_{i j}, i \in \mathcal{G} \\
D_{i} \omega_{i}=-F_{i}\left(n_{i}^{0} v_{i}+v_{i}^{0} n_{i}\right)-P_{L_{i}}-P_{i}^{\mathcal{L}}-\sum_{\substack{(i, j) \in \mathcal{E} \\
i \in \mathcal{L}}} T_{i j} \delta_{i j} \\
\dot{\delta}_{i j}=\omega_{i}-\omega_{j},(i \mathrm{~b}) \\
i, j) \in \mathcal{E}
\end{gathered}
$$




$$
\dot{P}_{M_{i}}=\frac{1}{T_{T G_{i}}}\left(P_{C_{i}}-P_{M_{i}}-\frac{\omega_{i}}{R_{i}}\right), i \in \mathcal{G}
$$

where $T_{i j}=v_{i}^{0} v_{j}^{0} B_{i j}>0,(i, j) \in \mathcal{E}$, and

$$
\begin{aligned}
P_{i}^{\mathcal{G}}= & P_{i}+\sum_{(i, j) \in \mathcal{E}, j \in \mathcal{L}} v_{i}^{0} B_{i j}\left(\delta_{i}^{0}-\delta_{j}^{0}\right) v_{j}, i \in \mathcal{G} \\
P_{i}^{\mathcal{L}}= & P_{i}+\sum_{(i, j) \in \mathcal{E}} v_{j}^{0} B_{i j}\left(\delta_{i}^{0}-\delta_{j}^{0}\right) v_{i} \\
& +\sum_{(i, j) \in \mathcal{E}, j \in \mathcal{L}} v_{i}^{0} B_{i j}\left(\delta_{i}^{0}-\delta_{j}^{0}\right) v_{j}, i \in \mathcal{L} .
\end{aligned}
$$

Let $\alpha_{i}=\delta_{i}-\delta_{m+n}, i \in \mathcal{G} \bigcup \mathcal{L} \backslash\{m+n\}, \omega=$ $\left[\omega_{1}, \cdots, \omega_{m+n}\right]^{T}, \alpha=\left[\alpha_{1}, \cdots, \alpha_{m+n-1}\right]^{T}$, and $\sigma=$ $\left[\sigma_{1}, \cdots, \sigma_{p}\right]^{T}$ where $\sigma_{k}=\delta_{i j}=\delta_{i}-\delta_{j}, i<j$ if the transmission line $(i, j) \in \mathcal{E}$ is indexed by $k$ (recall that the transmission lines are numbered $1, \cdots, p$ corresponding to a lexicographic ordering). Let $T=\operatorname{diag}\left\{T_{k}\right\} \in \mathbb{R}^{p \times p}$, where $T_{k}=T_{i j}$ if the line $(i, j) \in \mathcal{E}$ is indexed by $k$. Moreover, define these matrices to describe the interconnection structure of the network:

$$
\begin{aligned}
A_{0}= & {\left[a_{i k}\right]_{(m+n) \times p} } \\
= & \left\{\begin{array}{rr}
1 & \text { if bus } i \text { is connected to a bus } j>i \\
\text { through transmission line indexed by } k, \\
-1 & \text { if bus } i \text { is connected to a bus } j<i \\
\text { through transmission line indexed by } k,
\end{array}\right. \\
A= & {\left[\begin{array}{ll}
I_{m+n-1} & \mathbf{0}_{(m+n-1) \times 1}
\end{array}\right] \times A_{0} } \\
\Gamma_{1}= & {\left[\begin{array}{ll}
I_{m} & \mathbf{0}_{m \times(n-1)}
\end{array}\right]_{m \times(m+n-1)} } \\
\Gamma_{2}= & {\left[\begin{array}{ll}
\mathbf{0}_{(n-1) \times m} & I_{n-1} \\
-\mathbf{1}_{1 \times(m+n-1)}
\end{array}\right]_{n \times(m+n-1)} }
\end{aligned}
$$

Lemma 1 The following equalities hold:

$$
\begin{gathered}
A_{0}=\left[\begin{array}{c}
\Gamma_{1} \\
\Gamma_{2}
\end{array}\right] A \\
\sigma=A^{T} \alpha \\
\dot{\sigma}=A_{0}^{T} \omega .
\end{gathered}
$$

Proof: Note that $A_{0}$ is the incidence matrix of the directed graph $(\mathcal{G} \cup \mathcal{L}, \mathcal{E})$. So $\mathbf{1}_{1 \times(m+n)} A_{0}=\mathbf{0}_{1 \times p}$ holds, which means that (8a) is true (actually $A$ is the reduced incidence matrix resulting from $A_{0}$ ). Equations (8b) and $(8 \mathrm{c})$ can be verified from the definition above. A 6 -bus network in Figure 1 illustrates these relations.

The state-space version of Subsystem I is then given by

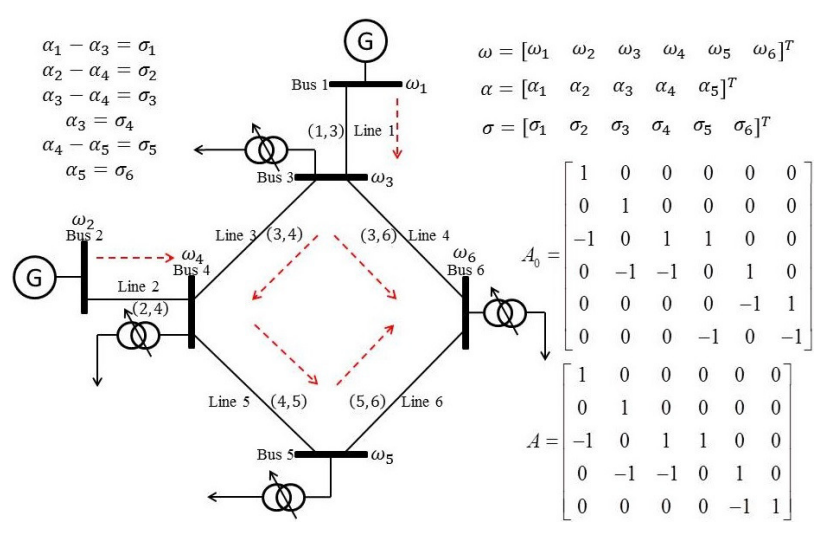

Fig. 1. A 6-bus network (the orientation is indicated by red dashed arrows).

$$
\begin{gathered}
M \dot{\omega}_{g}+D_{g} \omega_{g}=P_{M}-P^{\mathcal{G}}-\Gamma_{1} A T A^{T} \alpha \\
\dot{\alpha}=\Gamma_{1}^{T} \omega_{g}+\Gamma_{2}^{T} \omega_{l} \\
\omega_{l}=D_{l}^{-1}\left(-F\left(n_{T C}^{0} v_{l}+v_{l}^{0} n_{T C}\right)-P_{L}-P^{\mathcal{L}}\right. \\
\left.-\Gamma_{2} A T A^{T} \alpha\right) \\
\dot{P}_{M}=T_{T G}^{-1}\left(P_{C}-P_{M}-R^{-1} \omega_{g}\right)
\end{gathered}
$$

where $M=\operatorname{diag}\left\{M_{i}\right\} \in \mathbb{R}^{m \times m}, \omega_{g}=\left[\omega_{1}, \cdots, \omega_{m}\right]^{T}$, $D_{g}=\operatorname{diag}\left\{D_{1}, \cdots, D_{m}\right\}, P_{M}=\left[P_{M_{1}}, \cdots, P_{M_{m}}\right]^{T}$, $P^{\mathcal{G}}=\left[P_{1}^{\mathcal{G}}, \cdots, P_{m}^{\mathcal{G}}\right]^{T}, \omega_{l}=\left[\omega_{m+1}, \cdots, \omega_{m+n}\right]^{T}, D_{l}=$ $\operatorname{diag}\left\{D_{m+1}, \cdots, D_{m+n}\right\}, F=\operatorname{diag}\left\{F_{m+1}, \cdots, F_{m+n}\right\}$, $n_{T C}^{0}=\operatorname{diag}\left\{n_{m+1}^{0}, \cdots, n_{m+n}^{0}\right\}, v_{l}=\left[v_{m+1}, \cdots, v_{m+n}\right]^{T}$, $v_{l}^{0}=\operatorname{diag}\left\{v_{m+1}^{0}, \cdots, v_{m+n}^{0}\right\}, n_{T C}=\left[n_{m+1}, \cdots, n_{m+n}\right]^{T}$, $P_{L}=\left[P_{L_{m+1}}, \cdots, P_{L_{m+n}}\right]^{T}, P^{\mathcal{L}}=\left[P_{m+1}^{\mathcal{L}}, \cdots, P_{m+n}^{\mathcal{L}}\right]^{T}$, $T_{T G}=\operatorname{diag}\left\{T_{T G_{i}}\right\} \in \mathbb{R}^{m \times m}, P_{C}=\left[P_{C_{1}}, \cdots, P_{C_{m}}\right]^{T}$, and $R=\operatorname{diag}\left\{R_{i}\right\} \in \mathbb{R}^{m \times m}$.

Remark 3 Given $P_{C}, P_{L}, P^{\mathcal{G}}, P^{\mathcal{L}}$ and $n_{T C}^{0} v_{l}+v_{l}^{0} n_{T C}=$ $\mathbf{0}_{n \times 1}$, the equilibrium point of system (9) is uniquely determined as: $\omega_{g}^{*}=\nu \mathbf{1}_{m \times 1}$ where $\nu=\left(\mathbf{1}_{1 \times m}\left(P_{C}-\right.\right.$ $\left.\left.P^{\mathcal{G}}\right)-\mathbf{1}_{1 \times n}\left(P_{L}+P^{\mathcal{L}}\right)\right) /\left(\operatorname{tr}\left(D_{g}\right)+\operatorname{tr}\left(R^{-1}\right)+\operatorname{tr}\left(D_{l}\right)\right)$, $P_{M}^{*}=P_{C}-R^{-1} \omega_{g}^{*}$ and

$$
\begin{aligned}
& \alpha^{*}=\left(A T A^{T}\right)^{-1} \\
& \times\left[\begin{array}{c}
I_{m+n-1} \\
\mathbf{0}_{1 \times(m+n-1)}
\end{array}\right]^{T}\left[\begin{array}{c}
P_{C}-P^{\mathcal{G}}-\left(D_{g}+R^{-1}\right) \nu \mathbf{1}_{m \times 1} \\
-P_{L}-P^{\mathcal{L}}-D_{l} \nu \mathbf{1}_{n \times 1}
\end{array}\right]
\end{aligned}
$$

where $A T A^{T}$ is invertible [29]. On the other hand, given $P_{C}, P_{L}, P^{\mathcal{G}}, P^{\mathcal{L}}$ and $n_{T C}^{0} v_{l}+v_{l}^{0} n_{T C}=\mathbf{0}_{n \times 1}$, the equilibria of system (7) are determined as: $\omega_{i}^{*}=\nu, P_{M_{i}}^{*}=$ $P_{C_{i}}-R_{i}^{-1} \omega_{i}^{*}, i \in \mathcal{G}$, and

$$
\begin{aligned}
& A T \sigma^{*}= \\
& {\left[\begin{array}{c}
I_{m+n-1} \\
\mathbf{0}_{1 \times(m+n-1)}
\end{array}\right]^{T}\left[\begin{array}{c}
P_{C}-P^{\mathcal{G}}-\left(D_{g}+R^{-1}\right) \nu \mathbf{1}_{m \times 1} \\
-P_{L}-P^{\mathcal{L}}-D_{l} \nu \mathbf{1}_{n \times 1}
\end{array}\right] .}
\end{aligned}
$$

The above equation results in multiple solutions for $\sigma^{*}$ 
when the graph $(\mathcal{G} \cup \mathcal{L}, \mathcal{E})$ has mesh topology. However, all practical trajectories of system (7) converge to the unique equilibrium point $\left(\omega_{g}^{*}, A^{T} \alpha^{*}, P_{M}^{*}\right)[9]$ (Remark 5).

Subsystem II. Define $\epsilon=\operatorname{diag}\left\{\epsilon_{m+1}, \cdots, \epsilon_{m+n}\right\}, E=$ $\operatorname{diag}\left\{E_{m+1}, \cdots, E_{m+n}\right\}, Q_{L}=\left[Q_{L_{m+1}}, \cdots, Q_{L_{m+n}}\right]^{T}$, $Q=\left[Q_{m+1}, \cdots, Q_{m+n}\right]^{T}, T_{T}=\operatorname{diag}\left\{T_{T_{m+1}}, \cdots, T_{T_{m+n}}\right\}$, and a matrix $L$ as

$$
\begin{aligned}
L= & {\left[l_{i j}\right]_{n \times n} } \\
= & \left\{\begin{array}{c}
2 v_{m+i}^{0} B_{\mathrm{sh}, m+i}+\sum_{(m+i, k) \in \mathcal{E}}\left(2 v_{m+i}^{0}-v_{k}^{0}\right) B_{(m+i) k} \\
\text { when } m+i \in \mathcal{L}, i=j ; \\
-v_{m+i}^{0} B_{(m+i)(m+j)} \\
\text { when } m+i, m+j \in \mathcal{L}, i \neq j,(m+i, m+j) \in \mathcal{E} ; \\
0 \\
\text { when } m+i, m+j \in \mathcal{L}, i \neq j,(m+i, m+j) \notin \mathcal{E} .
\end{array}\right.
\end{aligned}
$$

Subsystem II can then be rearranged as

$$
\begin{gathered}
\dot{v}_{l}=-\left(\epsilon v_{l}^{0}\right)^{-1}\left(E\left(n_{T C}^{0} v_{l}+v_{l}^{0} n_{T C}\right)+Q_{L}+Q+L v_{l}\right) \\
\dot{n}_{T C}=-T_{T}^{-1}\left(n_{T C}^{0} v_{l}+v_{l}^{0} n_{T C}\right) .
\end{gathered}
$$

It should be emphasized that system (10) is applicable only for networks working around nominal states [23], and this is in accord with the situation considered.

Assumption $3 L$ is positive definite, i.e., $L \succ 0$.

This assumption is supported by the fact that the operating point determined by the ED problem should be stable and a sufficient condition for this is $L \succ 0[30,31]$.

Remark 4 In realistic power systems, nominal bus voltage magnitudes are within the interval $[\underline{v}, \bar{v}]$, e.g., $v_{i}^{0} \in[0.95$ p.u., 1.05p.u.] $( \pm 5 \%), i \in \mathcal{G} \cup \mathcal{L}$ [32], where p.u. means per unit. Nominal tap positions of OLTCs are within the interval $[\underline{n}, \bar{n}]$, e.g., $n_{i}^{0} \in$ [0.9p.u., 1.1p.u.] ( $\pm 10 \%), i \in \mathcal{L}[20,11]$. These operating ranges appear as constraints in the ED problem, e.g., the Optimal Power Flow (OPF) problem formulated in [33]. The voltage magnitude bounds $\underline{v}$ and $\bar{v}$ are usually close to 1 p.u. [34], which results in that all $v_{i}^{0}$ approximately equal [15]. When all $v_{i}^{0}$ are equal, $L \succ 0$ holds since it is strictly diagonal dominant. Note that the existence of shunt reactors at load buses actually increases diagonal elements in L. So Assumption 3 is not conservative.

Overview. The relationship between Subsystems I and II is illustrated in Figure 2. It can be seen that the output of Subsystem II affects I while there is no influence from I to II. This property simplifies the control design for the overall system: given constant bounded disturbance injection, if Subsystem II is asymptotically stable under

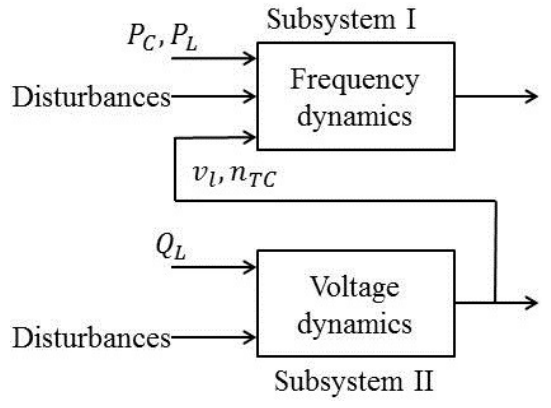

Fig. 2. System model overview.

the control input $Q_{L}$, the inputs $v_{l}, n_{T C}$ to Subsystem I can be regarded as constant when designing $P_{C}, P_{L}$.

\section{The Active Power Regulation Scheme}

Suppose that the network described by (9)-(10) is operating around a nominal state and that constant bounded disturbances $P_{i}, i \in \mathcal{G} \cup \mathcal{L}$ and $Q_{i}, i \in \mathcal{L}$ occur. Such disturbances bring real-time stress to the network, i.e., frequency and bus voltage magnitude/tap position deviations. It is expected that generators and loads can adjust their power generation and consumption $\left[P_{C}^{T}, P_{L}^{T}, Q_{L}^{T}\right]^{T}$ respectively in real-time using available information, not only to release the overall system from stress but also to result in more benefits. In this and the following sections, a real-time control scheme will be designed for generators and loads so that the following goals are achieved for the overall system: G1 - supply and demand are balanced at each bus; G2 - the aggregate power generation cost is minimized; G3 - the system transient performance is improved; G4 - bus voltage magnitude/tap position deviations are maintained within certain ranges; G5 - the generation control scheme is distributed, and the overall system is asymptotically stable regardless of communication delays; G6 - the load control scheme is completely decentralized in that users in each load/distribution network adjust their power consumption only based on local measurement.

\subsection{The Optimization Problem}

Consider the active power regulation problem using Subsystem I (9). Note that the terms relating to $v_{l}$ in $P^{\mathcal{G}}, P^{\mathcal{L}}$ and the term $F\left(n_{T C}^{0} v_{l}+v_{l}^{0} n_{T C}\right)$ come from Subsystem II while Subsystem I does not affect Subsystem II. Moreover, as will be shown in the next section, Subsystem II can be asymptotically stabilized and $F\left(n_{T C}^{0} v_{l}+v_{l}^{0} n_{T C}\right)=\mathbf{0}_{n \times 1}$ holds at steady state. So $v_{l}$ is regarded as constant (which leads to constant $P^{\mathcal{G}}, P^{\mathcal{L}}$ ) and $F\left(n_{T C}^{0} v_{l}+v_{l}^{0} n_{T C}\right)$ is set to $\mathbf{0}_{n \times 1}$ in this section. To achieve $\mathbf{G 1}$ and $\mathbf{G 2}$ above, formulate a steady-state optimization problem for generation control as

$$
\min _{P_{M}, \alpha} \sum_{i \in \mathcal{G}} C_{i}\left(P_{M_{i}}\right)
$$




$$
\begin{array}{cc}
\text { subject to } & P_{M}-P^{\mathcal{G}}-\Gamma_{1} A T A^{T} \alpha=\mathbf{0}_{m \times 1} \\
& -P^{\mathcal{L}}-\Gamma_{2} A T A^{T} \alpha=\mathbf{0}_{n \times 1}
\end{array}
$$

where $C_{i}\left(P_{M_{i}}\right)$ is the cost function for the generator, and $\left[P^{\mathcal{G} T}, P^{\mathcal{L} T}\right]^{T}$ is a given constant bounded disturbance (now the disturbance consists of two parts, resulting from exogenous disturbance injection and voltage variations respectively). Equations (11b)-(11c) ensure active power balance at each bus. Similar to [5], capacity constraints are not considered for generators since the network is working around a nominal state.

Remark 5 The reason why $P_{L}$ is not included in (11c) is the following: (i) the disturbance is expected to be cleared through only generation control, as in the standard tieline bias control [15]; (ii) according to [9], controllable loads can participate in primary frequency control under proportional actions, which could result in better transient performance (in accord with G3); (iii) the use of proportional control for loads leads to a completely decentralized scheme (in accord with $\boldsymbol{G} \boldsymbol{6}$ ). So $P_{L}$ has been excluded in (11c), and moreover, proportional actions for controllable loads will be used.

For problem (11), the following assumption is necessary.

Assumption 4 Each $C_{i}\left(P_{M_{i}}\right)$ is in quadratic form, i.e., $C_{i}\left(P_{M_{i}}\right)=\frac{1}{2} c_{i} P_{M_{i}}^{2}+b P_{M_{i}}, b \gg c_{i}>0, i \in \mathcal{G}$. Problem (11) is feasible.

This assumption is supported as follows. First, cost functions with quadratic form $C_{i}\left(P_{M_{i}}\right)=\frac{1}{2} c_{i} P_{M_{i}}^{2}+b_{i} P_{M_{i}}$, where $b_{i} \gg c_{i}>0$, are widely used for generators $[15,19]$. Note that the network is operating around a nominal state, i.e., for each generator, $P_{M_{i}}$ denotes a deviation from its nominal operating value $P_{M_{i}}^{0}$. If there is no disturbance, i.e., $\left[P^{\mathcal{G} T}, P^{\mathcal{L} T}\right]^{T}=\mathbf{0}_{(m+n) \times 1}$, the subsystem should stay at the nominal state so that $\left[P_{M}^{* T}, \alpha^{* T}\right]^{T}=$ $\mathbf{0}_{(2 m+n-1) \times 1}\left(P_{L}^{*}=\mathbf{0}_{n \times 1}\right.$ also holds $)$ is the optimal solution of problem (11). Since problem (11) is a convex problem with linear equality constraints, from its KKT conditions, $b_{i}=b_{j}, \forall i, j \in \mathcal{G}$ holds. Finally, the feasibility of the problem is a necessary assumption.

Equations (11b)-(11c) imply that $\mathbf{1}_{1 \times m}\left(P_{M}-P^{\mathcal{G}}\right)-$ $\mathbf{1}_{1 \times n} P^{\mathcal{L}}=0$ holds at steady state. Using proportional control for loads, $P_{L}=\mathbf{0}_{n \times 1}$ holds at steady state. These facts lead to $\omega_{g}=\mathbf{0}_{m \times 1}, P_{C}=P_{M}$ at steady state. Based on Remark 3 ( $\alpha$ can be uniquely derived given $\left.P_{C}, P_{L}=\mathbf{0}_{n \times 1}, P^{\mathcal{G}}, P^{\mathcal{L}}\right)$, problem (11) can be reformulated as

$$
\min _{P_{C}} \sum_{i \in \mathcal{G}} C_{i}\left(P_{C_{i}}\right)
$$

subject to $\mathbf{1}_{1 \times m}\left(P_{C}-P^{\mathcal{G}}\right)-\mathbf{1}_{1 \times n} P^{\mathcal{L}}=0$ regardless of $P_{M}, \alpha$. Under Assumption 4, the objective function becomes

$\sum_{i \in \mathcal{G}} \frac{1}{2} c_{i} P_{C_{i}}^{2}+b P_{C_{i}}=\sum_{i \in \mathcal{G}} \frac{1}{2} c_{i} P_{C_{i}}^{2}+b\left(\mathbf{1}_{1 \times m} P^{\mathcal{G}}+\mathbf{1}_{1 \times n} P^{\mathcal{L}}\right)$

which indicates that the choice of $b$ does not affect the optimal solution. Moreover, $c_{i}$ is chosen to be equal to $\tilde{c}_{i}$ in the global cost function of the generator at bus $i$, i.e., $\tilde{C}_{i}\left(\tilde{P}_{M_{i}}\right)=\frac{1}{2} \tilde{c}_{i} \tilde{P}_{M_{i}}^{2}+\tilde{b}_{i} \tilde{P}_{M_{i}}$, where $\tilde{b}_{i} \gg \tilde{c}_{i}>0, i \in \mathcal{G}$.

The goal is to design $P_{C}$ (recall Remark 1) so that it asymptotically stabilizes Subsystem I (9) where $v_{l}$ is constant and $F\left(n_{T C}^{0} v_{l}+v_{l}^{0} n_{T C}\right)=\mathbf{0}_{n \times 1}$, and the equilibria are the optimal solutions of the optimization problem $(11) /(12)$. This is presented next.

\subsection{Design Methodology}

For the optimization problem (12), since it is a strictly convex problem with a linear equality constraint, strong duality holds and the KKT conditions are necessary and sufficient conditions for optimality [35], given by

$$
\begin{gathered}
c_{i} P_{C_{i}}=c_{j} P_{C_{j}}, \forall i, j \in \mathcal{G} \\
\mathbf{1}_{1 \times m}\left(P_{C}-P^{\mathcal{G}}\right)-\mathbf{1}_{1 \times n} P^{\mathcal{L}}=0 .
\end{gathered}
$$

Equation (13a) holds for generator buses. Now extend this equality to all buses:

$$
c_{i} P_{C_{i}}=c_{j} P_{C_{j}},(i, j) \in \mathcal{E}
$$

where $c_{i}=1, i \in \mathcal{L}$ and $P_{C_{i}}, i \in \mathcal{L}$ are auxiliary variables. As will become clear later, the reason for introducing $c_{i} P_{C_{i}}, i \in \mathcal{L}$ is to keep communication only between neighbourhood buses.

Recall that Equation (3) is actually an integral controller which aims to drive $-B_{i} \omega_{i}-P_{\text {tie-line, } i}$ to zero. To keep the modification small, replace this term in the dynamics of $P_{C}$ by terms from the KKT conditions so that optimality is achieved at equilibrium:

$$
\begin{gathered}
\dot{P}_{C_{i}}=B_{i}\left(P_{M_{i}}-P_{C_{i}}\right)-K_{i} \sum_{(i, j) \in \mathcal{E}}\left(c_{i} P_{C_{i}}-c_{j} P_{C_{j}}\right), i \in \mathcal{G} \\
\dot{P}_{C_{i}}=-K_{i} \sum_{(i, j) \in \mathcal{E}}\left(c_{i} P_{C_{i}}-c_{j} P_{C_{j}}\right), i \in \mathcal{L}
\end{gathered}
$$

where the positions of $B_{i}, K_{i}>0$ have been changed since they are design parameters in (3). Compared to the conventional tie-line bias control which is decentralized, the above control scheme requires information exchange between neighbourhood buses as illustrated in Figure 3, which is distributed. The communication can be carried out via the Wide Area Network (WAN) [36]. 


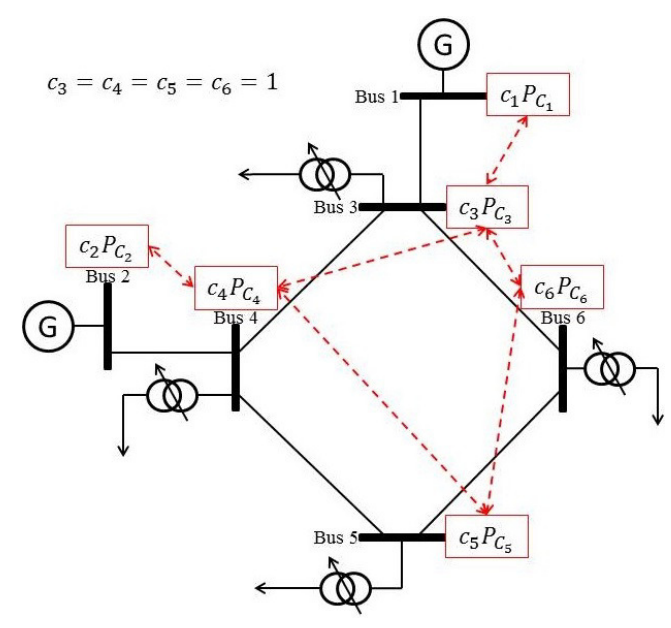

Fig. 3. Information exchange illustration for a 6-bus network.

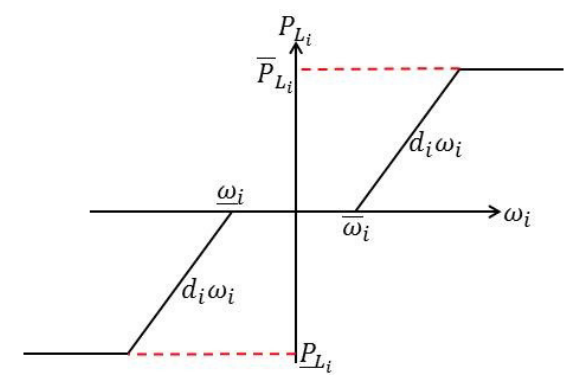

Fig. 4. $P_{L_{i}}=P_{L_{i}}\left(d_{i}, \underline{\omega}_{i}, \bar{\omega}_{i}, \underline{P}_{L_{i}}, \bar{P}_{L_{i}}\right), i \in \mathcal{L}$ with nonlinear characteristics, where the dead zone starts at $\underline{\omega}_{i}$ and ends at $\bar{\omega}_{i}$, and the saturation is inactive when $d_{i} \omega_{i} \in\left[\underline{P}_{L_{i}}, \bar{P}_{L_{i}}\right]$.

On the other hand, for controllable loads, proportional control is used to regulate them (recall Remark 5):

$$
P_{L_{i}}=P_{L_{i}}\left(d_{i}, \underline{\omega}_{i}, \bar{\omega}_{i}, \underline{P}_{L_{i}}, \bar{P}_{L_{i}}\right), i \in \mathcal{L}
$$

where $d_{i} \geq 0$ is the proportional gain and $P_{L_{i}}()$ is now a nonlinear function with dead zone (parameterized by $\underline{\omega}_{i}, \bar{\omega}_{i}, \underline{\omega}_{i} \leq 0 \leq \bar{\omega}_{i}$ ) and saturation (parameterized by $\left.\underline{P}_{L_{i}}, \bar{P}_{L_{i}}, \underline{P}_{L_{i}} \leq 0 \leq \bar{P}_{L_{i}}\right)$ characteristics, shown in Figure 4 . These loads actually add damping to the subsystem. Finally, the closed-loop subsystem dynamics in state-space form are given by

$$
\begin{gathered}
M \dot{\omega}_{g}+D_{g} \omega_{g}=P_{M}-P^{\mathcal{G}}-\Gamma_{1} A T A^{T} \alpha \\
\dot{\alpha}=\Gamma_{1}^{T} \omega_{g}+\Gamma_{2}^{T} D_{l}^{-1}\left(-P_{L}-P^{\mathcal{L}}-\Gamma_{2} A T A^{T} \alpha\right) \\
\dot{P}_{M}=T_{T G}^{-1}\left(P_{C}-P_{M}-R^{-1} \omega_{g}\right) \\
\dot{P}_{C}=B\left(P_{M}-P_{C}\right)-\left[\begin{array}{c}
I_{m} \\
\mathbf{0}_{n \times m}
\end{array}\right]^{T} K A_{0} A_{0}^{T}\left[\begin{array}{c}
C P_{C} \\
P_{C}^{\prime}
\end{array}\right] \\
\dot{P}_{C}^{\prime}=-\left[\begin{array}{c}
\mathbf{0}_{m \times n} \\
I_{n}
\end{array}\right]^{T} K A_{0} A_{0}^{T}\left[\begin{array}{c}
C P_{C} \\
P_{C}^{\prime}
\end{array}\right]
\end{gathered}
$$

where $B=\operatorname{diag}\left\{B_{i}\right\} \in \mathbb{R}^{m \times m}, K=\operatorname{diag}\left\{K_{M}, K_{N}\right\} \in$ $\mathbb{R}^{(m+n) \times(m+n)}, K_{M}=\operatorname{diag}\left\{K_{1}, \cdots, K_{m}\right\} \succ 0, K_{N}=$ $\operatorname{diag}\left\{K_{m+1}, \cdots, K_{m+n}\right\} \succ 0, C=\operatorname{diag}\left\{c_{i}\right\} \in \mathbb{R}^{m \times m}$, $P_{C}^{\prime}=\left[P_{C_{m}+1}, \cdots, P_{C_{m+n}}\right]^{T}$, and $P_{L}$ is given by $(14)$.

\subsection{Optimality, Stability and Delay Robustness}

Results relating to the optimality and stability of system (15) are first presented.

Theorem 1 Given a constant $\left[P^{\mathcal{G} T}, P^{\mathcal{L} T}\right]^{T}$, under Assumption 4, the equilibrium point of system (15) is unique and $P_{C}^{*}$ is the optimal solution of problem (12) $\left(\left[P_{M}^{* T}, \alpha^{* T}\right]^{T}\right.$ is the optimal solution of problem (11)).

Proof: See the Appendix.

Theorem 2 Given a constant $\left[P^{\mathcal{G} T}, P^{\mathcal{L} T}\right]^{T}$, under Assumption 4, starting from any feasible initial point, the trajectories generated by system (15) converge to its unique equilibrium point if $K_{M}=\kappa C R^{-1} B$ and $K_{N}=\kappa I_{n}$ hold where $\kappa>0$.

Proof: See the Appendix.

As described previously, the implementation of the proposed generation control requires communication between neighbourhood buses carried out via the WAN. So delays could occur in the network. Now consider the case that the subsystem is operating under communication delays, given by $\tau_{j i}>0$ if a signal is passed from bus $j$ to bus $i$. System (15) then becomes

$$
\begin{aligned}
M \dot{\omega}_{g}(t)= & -D_{g} \omega_{g}(t)+P_{M}(t)-P^{\mathcal{G}}-\Gamma_{1} A T A^{T} \alpha(t) \\
\dot{\alpha}(t)= & \Gamma_{1}^{T} \omega_{g}(t)+\Gamma_{2}^{T} D_{l}^{-1}\left(-P_{L}(t)-P^{\mathcal{L}}\right. \\
\left.-\Gamma_{2} A T A^{T} \alpha(t)\right) & (16 \mathrm{a}) \\
\dot{P}_{M}(t)= & T_{T G}^{-1}\left(P_{C}(t)-P_{M}(t)-R^{-1} \omega_{g}(t)\right) \\
\dot{P}_{C_{i}}(t)= & B_{i}\left(P_{M_{i}}(t)-P_{C_{i}}(t)\right) \\
& \left.-K_{i} \sum_{(i, j) \in \mathcal{E}}\left(c_{i} P_{C_{i}}(t)-c_{j} P_{C_{j}}\left(t-\tau_{j i}\right)\right), i \in \mathcal{G}\right)
\end{aligned}
$$

$$
\dot{P}_{C_{i}}(t)=-K_{i} \sum_{(i, j) \in \mathcal{E}}\left(c_{i} P_{C_{i}}(t)-c_{j} P_{C_{j}}\left(t-\tau_{j i}\right)\right), i \in \mathcal{L}
$$

where $P_{C_{i}}(\theta)=\phi_{i}(\theta), \theta \in[-h, 0], \phi_{i} \in \mathcal{C}_{1, h}, i \in \mathcal{G} \bigcup \mathcal{L}$ and $h=\max _{(i, j) \in \mathcal{E}}\left\{\max \left\{\tau_{i j}, \tau_{j i}\right\}\right\}$. Note that $\tau_{i j}=\tau_{j i}$ is not required here. The following theorem shows the stability of system (16).

Theorem 3 Given a constant $\left[P^{\mathcal{G} T}, P^{\mathcal{L} T}\right]^{T}$, under Assumption 4 , the equilibrium point of system (16) is unique 
and asymptotically stable if $K_{M}=\kappa C R^{-1} B$ and $K_{N}=$ $\kappa I_{n}$ hold where $\kappa>0$. Moreover, $P_{C}^{*}$ is the optimal solution of problem (12).

Proof: See the Appendix.

Theorem 3 indicates that the asymptotic stability of (16) is independent of delays (so $\mathbf{G 5}$ is achieved) by choosing $K_{M}=\kappa C R^{-1} B$ and $K_{N}=\kappa I_{n}$ where $\kappa>0$. This is also the nominal stability condition given in Theorem 2 .

\section{The Reactive Power Regulation Scheme}

In this section, the reactive power regulation problem using Subsystem II (10) is considered. A method to detect tap changer variations of OLTCs is proposed so that primary-side voltage magnitude deviations can be detected at end users in each load. Therefore, the overall load control scheme can be designed in a completely decentralized manner (in accord with G6) and bus voltage magnitude/tap position deviations can be maintained within certain ranges by controlling reactive power consumption (in accord with G4).

\subsection{Design Methodology and Stability}

As discussed in Remark 4, primary-side voltage magnitudes and tap positions of OLTCs should be within certain intervals to ensure secure and stable operation. When a constant bounded disturbance $Q$ occurs and there are bus voltage magnitude/tap position deviations close to or out of permitted ranges, users (active voltage control devices) need to adjust their reactive power consumption so that the system stays away from dangerous operating areas. To achieve this, simple integral actions can be introduced for controllable loads given by

$$
\begin{gathered}
Q_{L_{i}}=Q_{L_{i}}^{+}-Q_{L_{i}}^{-}, i \in \mathcal{L} \\
\dot{Q}_{L_{i}}^{+}=h_{i}^{+}\left(v_{i}-\bar{v}_{i}\right)_{0}^{\bar{Q}_{L_{i}}}, i \in \mathcal{L} \\
\dot{Q}_{L_{i}}^{-}=h_{i}^{-}\left(-v_{i}+\underline{v}_{i}\right)_{0}^{-\underline{Q}}{ }_{L_{i}}, i \in \mathcal{L}
\end{gathered}
$$

where $h_{i}^{+}, h_{i}^{-}>0$ are controller gains, and the permitted range for $v_{i}$ is $\left[\underline{v}_{i}, \bar{v}_{i}\right], \underline{v}_{i} \leq 0 \leq \bar{v}_{i}, \underline{v}_{i} \neq \bar{v}_{i}$. These bounds depend on both the voltage magnitude deviation limit and the tap position deviation limit. For example, if $v_{i}^{0}=n_{i}^{0}=1$ p.u., and $\pm 10 \%$ voltage magnitude deviation and $\pm 5 \%$ tap position deviation are allowed for OLTC $i$, then $\left[\underline{v}_{i}, \bar{v}_{i}\right]=[-0.05$ p.u., 0.05p.u. $]$ since $n_{i}^{0} v_{i}+v_{i}^{0} n_{i}=0$ holds at steady state. Moreover, each $Q_{L_{i}}$ has a capacity constraint given by $Q_{L_{i}} \in\left[\underline{Q}_{L_{i}}, \bar{Q}_{L_{i}}\right]$ where $\underline{Q}_{L_{i}} \leq 0 \leq \bar{Q}_{L_{i}}$. When $v_{i}>\bar{v}_{i}$ which indicates that the primary-side voltage magnitude of the OLTC at bus $i$ is above its nominal operation range, the controllable part of load $i$ increases its reactive power con- sumption, i.e., $Q_{L_{i}}^{+}$increases and $Q_{L_{i}}$ goes up $\left(Q_{L_{i}}^{-}\right.$decreases to zero), or vice versa. The designed dynamics $\dot{Q}_{L_{i}}^{+}, \dot{Q}_{L_{i}}^{-}$are similar to those commonly used in Internet congestion control problems [37].

Let $Q_{L}^{+}=\left[Q_{L_{m+1}}^{+}, \cdots, Q_{L_{m+n}}^{+}\right]^{T}, H^{+}=\operatorname{diag}\left\{h_{m+1}^{+}, \cdots\right.$, $\left.h_{m+n}^{+}\right\}, \bar{v}=\left[\bar{v}_{m+1}, \cdots, \bar{v}_{m+n}\right]^{T}, \bar{Q}_{L}=\left[\bar{Q}_{L_{m+1}}, \cdots\right.$, $\left.\bar{Q}_{L_{m+n}}\right]^{T}, Q_{L}^{-}=\left[Q_{L_{m+1}}^{-}, \cdots, Q_{L_{m+n}}^{-}\right]^{T}, H^{-}=\operatorname{diag}\left\{h_{m+1}^{-}\right.$, $\left.\cdots, h_{m+n}^{-}\right\}, \underline{v}=\left[\underline{v}_{m+1}, \cdots, \underline{v}_{m+n}\right]^{T}, \underline{Q}_{L}=\left[\underline{Q}_{L_{m+1}}, \cdots\right.$, $\left.\underline{Q}_{L_{m+n}}\right]^{T}$. The state-space description of Subsystem II (10) with controller (17) is

$$
\begin{array}{rr}
\dot{v}_{l}=-\left(\epsilon v_{l}^{0}\right)^{-1}\left(E\left(n_{T C}^{0} v_{l}+v_{l}^{0} n_{T C}\right)+Q_{L}^{+}-Q_{L}^{-}+Q+L v_{l}\right) \\
\dot{n}_{T C}=-T_{T}^{-1}\left(n_{T C}^{0} v_{l}+v_{l}^{0} n_{T C}\right) & (18 \mathrm{a}) \\
\dot{Q}_{L}^{+}=H^{+}\left(v_{l}-\bar{v}\right)_{\mathbf{0}_{n \times 1}} & (18 \mathrm{c}) \\
\dot{Q}_{L}^{-}=H^{-}\left(-v_{l}+\underline{v}\right)_{\mathbf{o}_{n \times 1}}^{-Q_{L}} & (18 \mathrm{~d})
\end{array}
$$

where Equations (18c)-(18d) are vector forms of Equations $(17 b)-(17 c)$.

Theorem 4 Given a constant $Q$, under Assumption 3, the equilibrium point of system (18) is unique and asymptotically stable.

Proof: See the Appendix.

Remark 6 When there are buses whose voltage magnitude deviations are out of permitted ranges, and the controllable reactive parts of loads at these buses reach maxima/minima, the network will be working at abnormal states. To avoid this and keep the network safe, these buses need to shed loads [20], e.g., to change $\tilde{Q}_{V_{i}}$ (change $\left.\tilde{q}_{i}\right)$ in Equation (5b).

Theorem 4 shows the asymptotic stability of Subsystem II under the reactive power regulation scheme (17). The controller requires users to measure the primaryside voltage magnitude deviation of the OLTC at the bus they are connected to. This deviation can be derived for end users by detecting the tap position deviation of the OLTC and using $(18 \mathrm{~b}) /(6 \mathrm{c})$, provided with extra information: $T_{T_{i}}$ which is constant, and $n_{i}^{0}, v_{i}^{0}$ which result from the ED problem at a more slower time-scale and are fixed during a certain period of time. An approach to detect tap position variations is presented next so that the proposed decentralized controller is applicable.

\subsection{Tap Position Deviation Detection}

Root Mean Square (RMS) voltage measurement has been used to classify system events such as induction motor starting, voltage step change, transformer saturation, voltage swell, etc. [38]. In this paper, the RMS 
voltage that is derived from the mains voltage measurement, is used to determine the tap change event of the OLTC. Tap position variations can then be determined through the tap change detection. As one cycle RMS voltage suffers from precision issue during event classification [38], half cycle RMS voltage is used.

In order to determine the tap change event, a training data set must be provided, which was obtained from the local Distribution Network Operator (DNO) ${ }^{2}$. Tap change information, including tap time and tap position of the OLTC, is provided and used to form a basis of the training data set. Information on the voltage change caused by other events, such as induction motor starting and transformer saturation, is used to form the rest of the training data set.

The training data set is then divided into two subsets for training and validation respectively. Voltage features are investigated to determine the tap change event and the detection approach uses seven features as illustrated in Figure 3 in [39]. A decision tree is then used as the classifier so that only a voltage change with all its seven features within certain ranges is classified as a tap change. Further details of the features and classification performance can be found in [39].

Tap position variations can be inferred once tap change events are determined. A typical profile of the variation detection during a tap transit is shown in Figure 5. It can be seen from the plot that a tap change event takes about 50 milliseconds to complete. To achieve real-time tap position variation detection, any potential voltage change needs to be extracted to go through the determination approach. A detection index given by

$$
\mathcal{D I}(i)=\left|V_{r m s}(i+6)-V_{r m s}(i)\right|
$$

which is obtained by calculating the difference between two RMS voltages $\left(V_{r m s}\right)$ in the distance of six half cycles, is used for this purpose, where $i$ indicates the $i^{\text {th }}$ half cycle sampling point. A threshold $\eta$ can be obtained from the local DNO for each sampling location so that if $\mathcal{D I}(i) \geq \eta$ is true, the voltage change between the $i^{\text {th }}$ point and the $(i+6)^{\text {th }}$ point is potentially caused by a tap change. Otherwise, the voltage change between the $i^{\text {th }}$ point and the $(i+6)^{\text {th }}$ point is not caused by a tap change. A sliding window is created to calculate $\mathcal{D} \mathcal{I}(i)$ at each newly generated half cycle RMS voltage, which is then compared with $\eta$ to determine if the $i^{\text {th }}$ voltage point is a potential start of a tap change event.

Under the proposed detection approach, the overall load control scheme given by Equations (14) and (17) is completely decentralized. Provided with information about

\footnotetext{
${ }^{2}$ In this work, a collaboration was established with the local DNO, Scottish \& Southern Energy plc (SSE).
}
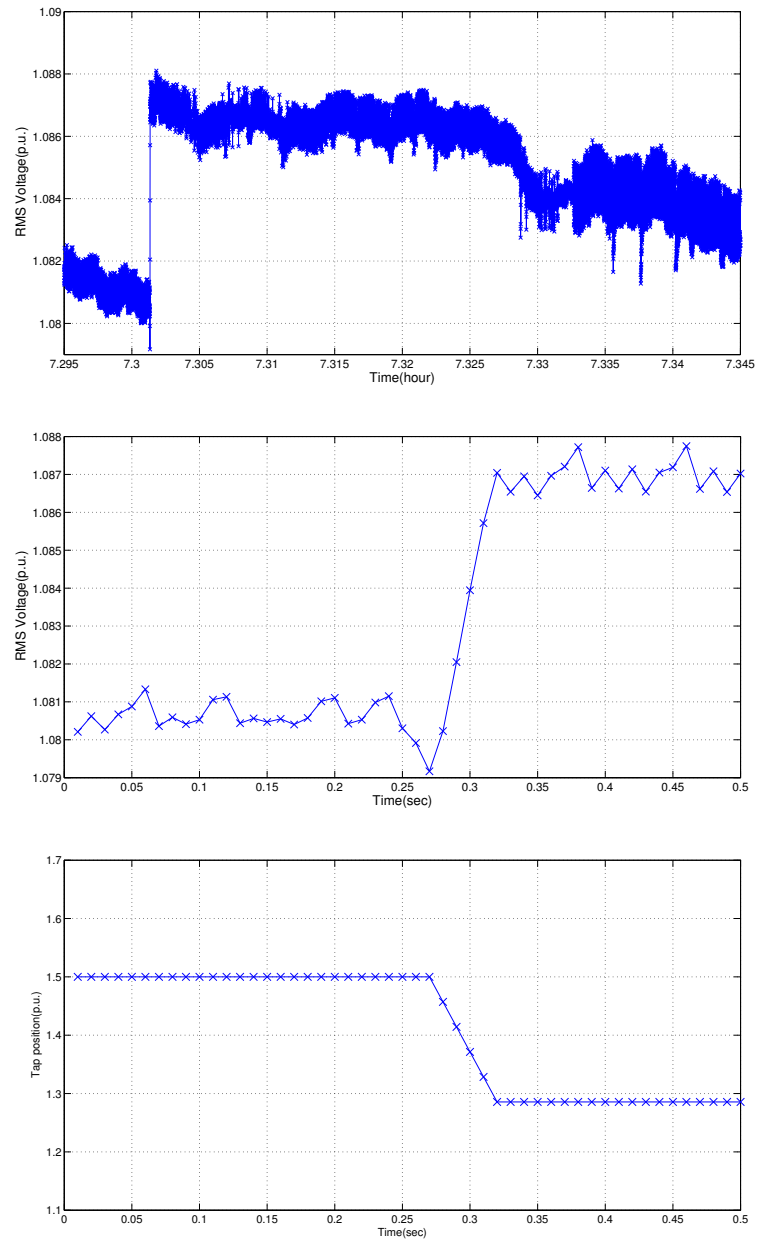

Fig. 5. Tap variation detection. Top: the voltage in longer time span where a tap change event occurred. Middle: the voltage transient during the tap change event. Bottom: the detected tap position variation within the same period (the information about the tap and the nominal state is given).

nominal states which updates at the same time-scale as solving the ED problem, each user only needs to measure the frequency and mains voltage locally for power regulation. This does not require any real-time communication and can be easily implemented.

\section{$5 \quad$ Numerical Investigations}

Numerical investigations for the 6-bus network illustrated in Figure 1 are presented in this section. Table 1 shows the parameter values of the overall system. Besides constant (step change) exogenous disturbances, time-varying disturbances are considered as given in Figure 6 (bottom two subfigures). Under these disturbances, generators and loads use the control scheme summarized in the next page to regulate their power generation and consumption. The simulation results are shown in Figures 6-7. Since changing $d_{i}$ does not affect Subsystem II, 
Table 1

Parameters of the overall system

\begin{tabular}{|c|c|c|c|}
\hline Parameter & Value (p.u.) & Parameter & Value (p.u.) \\
\hline$M_{i}$ & 5,4 & $D_{i}$ & $2,1.5,1,0.8,0.3,0.5$ \\
\hline$T_{T G_{i}}$ & 5,6 & $F_{i}$ & $1.5,0.6,3,2$ \\
\hline All $R_{i}$ & 0.05 & $B_{i j}$ & $24,25,23,26,27,22$ \\
\hline All $B_{\mathrm{sh}, i}$ & 2 & $E_{i}$ & $1.5,3,2.2,5$ \\
\hline All $v_{i}^{0}$ & 1 & $n_{i}^{0}$ & $1.1,1.05,0.95,1$ \\
\hline$\delta_{i}^{0}, i \in \mathcal{G}$ & $0.5,0.4$ & $\delta_{i}^{0}, i \in \mathcal{L}$ & $0.4,0.3,0.2,0.3$ \\
\hline All $B_{i}$ & 5 & $c_{i}$ & $0.1,0.2$ \\
\hline$\kappa$ & 2 & $T_{T_{i}}$ & $0.4,0.2,0.7,0.5$ \\
\hline All $\epsilon_{i}$ & 0.1 & All $_{i}^{+}, h_{i}^{-}$ & 10 \\
\hline All $\underline{\omega}_{i}, \bar{\omega}_{i}$ & 0 & $\bar{P}_{L_{i}}=-\underline{P}_{L_{i}}$ & $1,0.5,0.8,0.6$ \\
\hline All $\bar{v}_{i}=-\underline{v}_{i}$ & 0.02 & $\bar{Q}_{L_{i}}=-\underline{Q}_{L_{i}}$ & $0.5,0.3,1,0.7$ \\
\hline
\end{tabular}

\section{Control scheme (in continuous time)}

\section{Generator side:}

$$
\begin{aligned}
& \dot{P}_{C_{i}}=B_{i}\left(P_{M_{i}}-P_{C_{i}}\right)-K_{i} \sum_{(i, j) \in \mathcal{E}}\left(c_{i} P_{C_{i}}-c_{j} P_{C_{j}}\right), i \in \mathcal{G} \\
& \dot{P}_{C_{i}}=-K_{i} \sum_{(i, j) \in \mathcal{E}}\left(c_{i} P_{C_{i}}-c_{j} P_{C_{j}}\right), i \in \mathcal{L} \text { (auxiliary states) }
\end{aligned}
$$

\section{Load side:}

$$
\begin{gathered}
P_{L_{i}}=P_{L_{i}}\left(d_{i}, \underline{\omega}_{i}, \bar{\omega}_{i}, \underline{P}_{L_{i}}, \bar{P}_{L_{i}}\right), i \in \mathcal{L} \\
Q_{L_{i}}=Q_{L_{i}}^{+}-Q_{L_{i}}^{-}, i \in \mathcal{L} \\
\dot{Q}_{L_{i}}^{+}=h_{i}^{+}\left(-\left(n_{i}^{0}\right)^{-1}\left(T_{T_{i}} \dot{n}_{i}+v_{i}^{0} n_{i}\right)-\bar{v}_{i}\right)_{0}^{\bar{Q}_{L_{i}}}, i \in \mathcal{L} \\
\dot{Q}_{L_{i}}^{-}=h_{i}^{-}\left(\left(n_{i}^{0}\right)^{-1}\left(T_{T_{i}} \dot{n}_{i}+v_{i}^{0} n_{i}\right)+\underline{v}_{i}\right)_{0}^{-\underline{Q}_{L_{i}}}, i \in \mathcal{L}
\end{gathered}
$$

where the function $P_{L_{i}}()$ is shown in Figure 4. Each generator/generator bus locally measures $P_{M_{i}}$ and sends $c_{i} P_{C_{i}}$ to its neighbours, and each load bus sends $c_{i} P_{C_{i}}$ to its neighbours, as illustrated in Figure 3. Given local information $T_{T_{i}}, n_{i}^{0}, v_{i}^{0}$, each end user locally measures $\omega_{i}$ and detects $n_{i}$ using the method proposed in Section 4.2

the response of bus voltages and load reactive power in the cases when $d_{i}=5$ and $d_{i}=15$ is the same as for $d_{i}=0$.

From Figure 6, it can be seen that under disturbances, the proposed control scheme releases the network from stress, and moreover G1, G2 and G4 are achieved. Comparing Figures 6 and 7 , it is true that the introduction of controllable loads improves the transient behaviour of the overall system, i.e., G3 is achieved. As the proportional gain $d_{i}$ (a common proportional gain has been used in this example) increases, the oscillations and overshoots in the response of bus frequency and generator mechanical power decrease, as illustrated in Figure 7. Next, the same scenario under a homogeneous delay, i.e., all $\tau_{i j}=20 \mathrm{~s}$, is considered. The simulation results are shown in Figure 8. After adding delays, the response speed slows down, while the trajectories are similar to
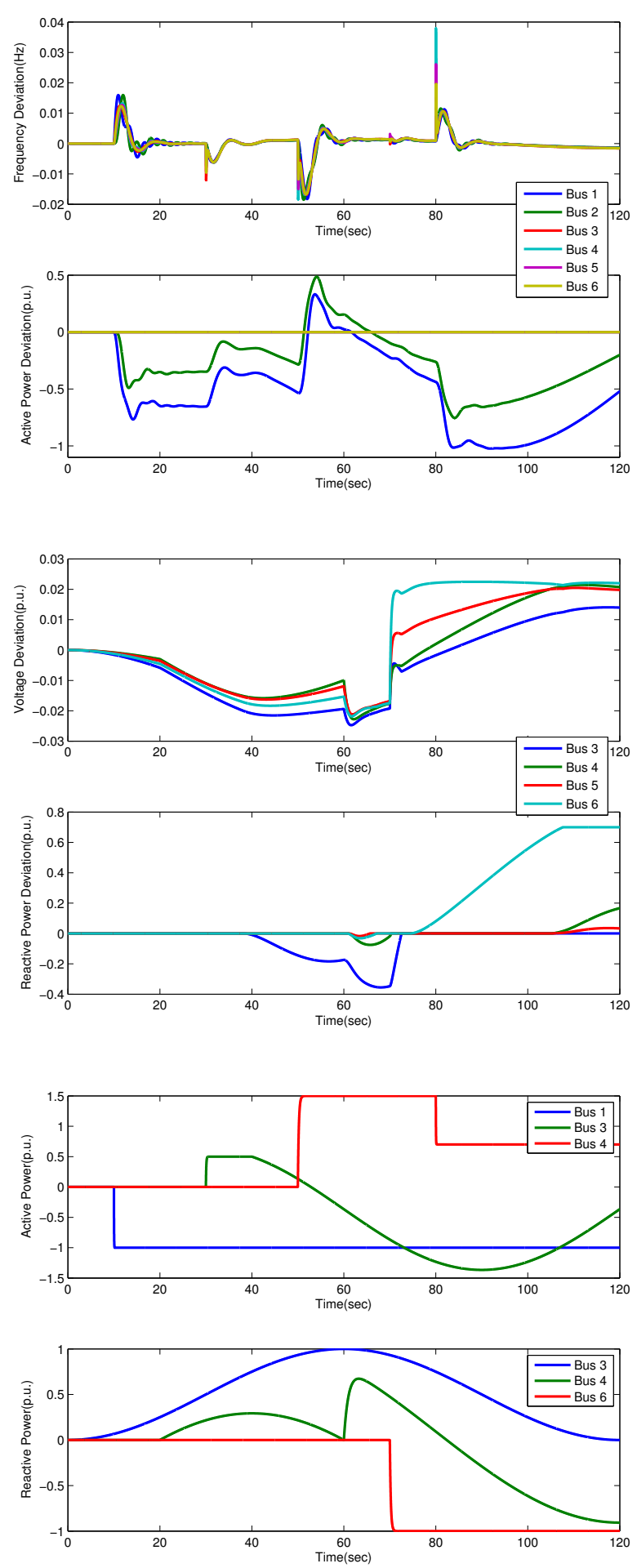

Fig. 6. System response with the proposed control scheme where all $d_{i}=0$. Top two: frequency at each bus, mechanical power of generators and active power of controllable loads. Middle two: voltage at each load bus and reactive power of controllable loads. Bottom two: active and reactive parts of disturbances at selected buses. 

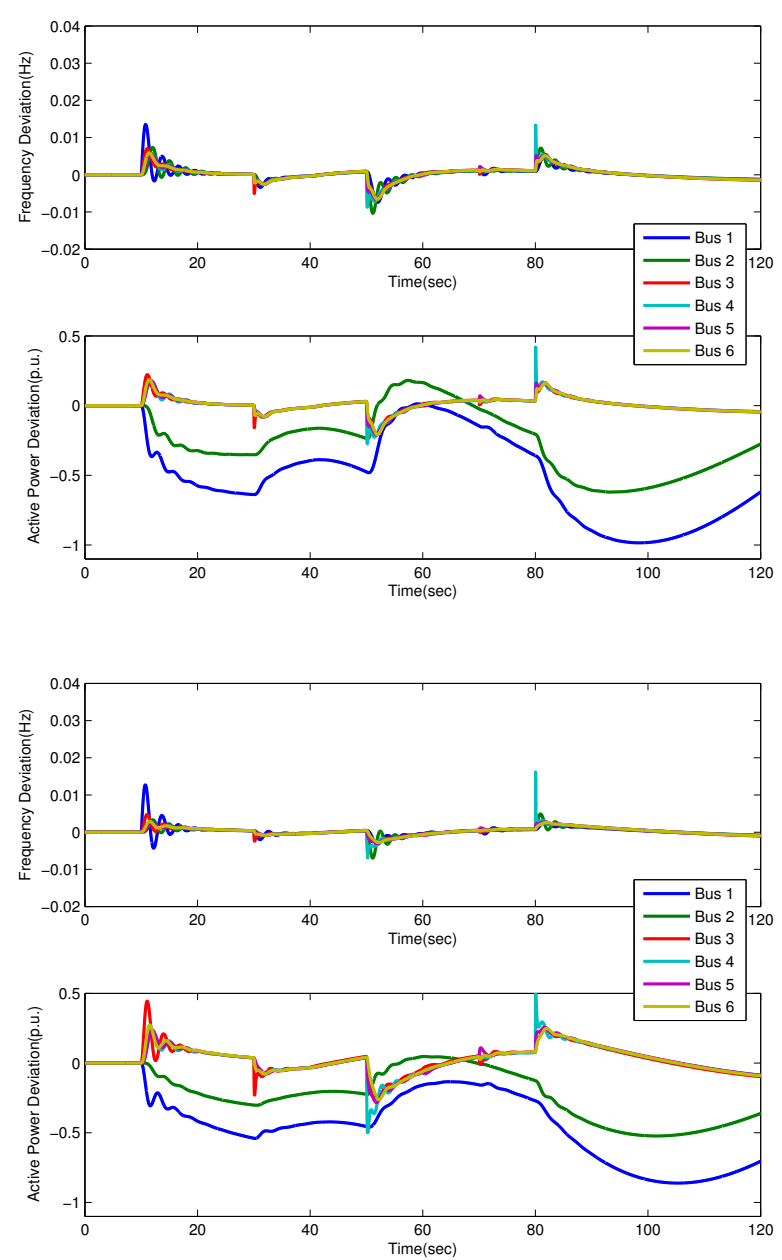

Fig. 7. System response with the proposed control scheme (the range of $y$-axis is the same as in Figure 6). Top two: all $d_{i}=5$. Bottom two: all $d_{i}=15$.

those in the undelayed case. Since adding delays does not affect Subsystem II, the response of bus voltages and load reactive power is the same as in the undelayed case.

\section{Conclusion and Future Work}

In this paper, a real-time distributed generation control scheme and a real-time decentralized load control scheme have been designed for power networks with tapchanging transformers. Under appropriate assumptions, i.e., Assumptions 1-2, the state-space description of a conventional power network model operating around a nominal state was first obtained, where the system was decomposed into two subsystems, i.e., frequency dynamics and voltage dynamics. For Subsystem I describing frequency dynamics, an optimization problem relating to generator active power regulation under exogenous disturbances was formulated. A distributed controller was proposed and the optimality, stability and delay robustness of the controlled subsystem was studied. Moreover,

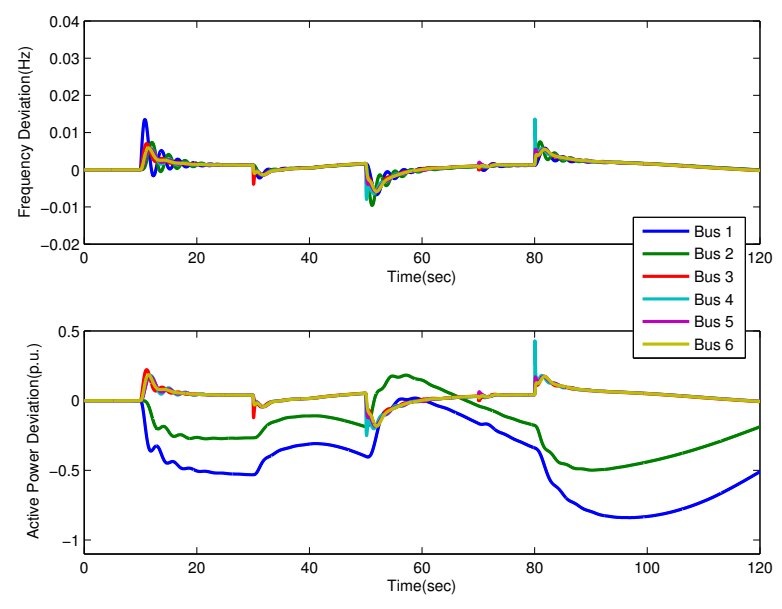

Fig. 8. System response with the proposed control scheme under time delays $\left(\tau_{i j}=20 \mathrm{~s}\right)$, where all $d_{i}=5$ (the range of $\mathrm{y}$-axis is the same as in Figure 6).

nonlinear proportional actions were introduced for controllable loads to improve the performance of the overall subsystem, which was demonstrated by numerical examples in Section 5. For Subsystem II describing voltage dynamics, under a mild assumption, i.e., Assumption 3 , simple integral control with projection characteristics was introduced for controllable loads and the asymptotic stability of the closed-loop subsystem was shown. A method for end users in each load to detect the tap position variation of the OLTC at the bus they are connected to was proposed, thus, the load control scheme is completely decentralized and can be applied easily. For the overall system (Subsystems I and II), numerical experiments illustrated the good performance of the proposed controller. To conclude, with the designed generator and load control scheme, the overall system can be released from stress resulting from disturbances, e.g. variations in both supply and demand, and renewable energy injections, and in the meantime achieve benefits G1-G6 shown at the beginning of Section 3.

The key contribution of this paper is summarized as follows. First, the proposed generation control scheme, which asymptotically stabilizes the frequency dynamics regardless of communication delay (there is very little information exchange) and minimizes aggregate power generation cost, can be used as a redesigned generation control scheme/tie-line bias control at control area levels (recall Remark 1). Moreover, the proposed load control scheme, which consists of proportional actions in real power regulation and integral actions in reactive power regulation, is decentralized, tractable and simple for realization. Lastly, the proposed tap change detection approach can be used to estimate the tap position variation. It does not require any real-time communication, and needs only local voltage measurement, plus information about nominal states which is not updated fre- 
quently (usually at the same time-scale as solving the ED problem to determine the nominal operating point).

This paper is the first step towards designing control and optimization schemes for power networks in real-time, i.e., merging and redesigning conventional primary and secondary control for power systems. Future work will focus on more detailed models with more complexity. By removing Assumption 1, voltage dynamics at generator buses will be considered where the effect of flux decay can be included. By removing Assumption 2, the physical coupling between active power flows and bus voltage magnitudes, and between reactive power flows and bus angle differences will be considered. This is especially important for subtransmission level networks where bus voltage is below $33 \mathrm{kV}$, since the coupling becomes strong in these networks. Also, deriving more general voltage stability conditions for the closed-loop system than those given by Assumption 3 is an interesting topic, where Sum of Squares (SOS) techniques [40] can be used. In addition, the choice of design parameters, i.e., $B, K, H^{+}, H^{-}$and $d_{i}, i \in \mathcal{L}$, will be investigated in order to bring added robustness to the overall system.

\section{References}

[1] A. Jokic. Price-based optimal control of electrical power systems. PhD dissertation, Department of Electrical Engineering, Eindhoven University of Technology, 2007.

[2] X. Zhang and A. Papachristodoulou. A real-time control framework for smart power networks with star topology. In Proc. of 2013 American Control Conference, pages 50625067, 2013.

[3] X. Zhang and A. Papachristodoulou. Distributed dynamic feedback control for smart power networks with tree topology. In Proc. of 2014 American Control Conference, pages 11561161,2014

[4] M. Andreasson, D. V. Dimarogonas, K. H. Johansson, and H. Sandberg. Distributed vs. centralized power systems frequency control under unknown load changes. In Proc. European Control Conference, pages 3524-3529, 2013.

[5] N. Li. Distributed optimization in power networks and general multi-agent systems. PhD dissertation, California Institute of Technology, 2013.

[6] S. You and L. Chen. Reverse and forward engineering of frequency control in power networks. In Proc. 53rd IEEE Conference on Decision and Control, pages 191-198, 2014.

[7] A. Kiani and A. Annaswamy. A hierarchical transactive control architecture for renewables integration in smart grids. In Proc. 51st IEEE Conference on Decision and Control, pages 4985-4990, 2012.

[8] F. Dörfler, J. W. Simpson-Porco, and F. Bullo. Breaking the hierarchy: Distributed control and economic optimality in microgrids. IEEE Transactions on Control of Network Systems, 2014, arXiv:1401.1767[math.OC].

[9] C. Zhao, U. Topcu, N. Li, and S. Low. Design and stability of load-side primary frequency control in power systems. IEEE Transactions on Automatic Control, 59(5):1177-1189, 2014.

[10] E. Mallada and S. H. Low. Distributed frequency-preserving optimal load control. In IFAC World Congress, pages 54115418,2014
[11] K. T. Vu, C. C. Liu, C. W. Taylor, and K. M. Jimma. Voltage instability: Mechanisms and control strategies. Proc. IEEE, 83(11):1442-1455, 1995.

[12] D. Callaway and I. Hiskens. Achieving controllability of electric loads. Proc. IEEE, 99(1):184-199, 2011.

[13] J. A. Short, D. G. Infield, and L. L. Ferris. Stabilization of grid frequency through dynamic demand control. IEEE Transactions on Power Systems, 23(3):1284-1293, 2007.

[14] A. R. Bergen and D. J. Hill. A structure preserving model for power system stability analysis. IEEE Transactions on Power Apparatus and Systems, 100(1):25-35, 1981.

[15] A. R. Bergen and V. Vittal. Power Systems Analysis. Pearson Education Inc. and Dorling Kindersley Publishing Inc., second edition, 2000.

[16] N. Narasimhamurthi and M. T. Musavi. A generalized energy function for transient stability analysis of power systems. IEEE Transactions on Circuits and Systems, CAS-31:637645, 1984.

[17] C. C. Liu and K. T. Vu. Analysis of tap-changer dynamics and construction of voltage stability regions. IEEE Transactions on Circuits and Systems, 36(4):575-590, 1989.

[18] M. Hong, C. C. Liu, and M. Gibescu. Complete controllability of an n-bus dynamic power system model. IEEE Transactions on Circuits and Systems-I, 46(6):700713, 1999.

[19] F. L. Alvarado, J. Meng, C. DeMarco, and W. Mota. Stability analysis of interconnected power systems coupled with market dynamics. IEEE Transactions on Power Systems, 16(4):695-701, 2001.

[20] P. Kundur. Power System Stability and Control. New York: McGraw-Hill, 1994.

[21] D. Kirschen and G. Strbac. Fundamentals of Power System Economics. John Wiley \& Sons, Ltd, first edition, 2004.

[22] J. W. Simpson-Porco, F. Dörfler, and F. Bullo. Voltage stabilization in microgrids via quadratic droop control. In Proc. 52nd IEEE Conference on Decision and Control, pages 7582-7589, 2013.

[23] A. R. Bergen, D. J. Hill, and C. L. DeMarco. A Lyapunov function for multimachine power systems with generator flux decay and voltage dependent loads. Int. J. Elect. Power Energy Syst., 8(1):2-10, 1986.

[24] C. A. Cañizares. Voltage collapse and transient energy function analysis of $\mathrm{AC} / \mathrm{DC}$ systems. PhD dissertation, University of Wisconsin-Madison, 1991.

[25] C. L. DeMarco and A. R. Bergen. A security measure for random load disturbances in nonlinear power system models. IEEE Transactions on Circuits and Systems, CAS34(12):1546-1557, 1987.

[26] G. Andersson. Modelling and analysis of electric power systems. In Technical Report, ETH Zurich, 2008.

[27] G. Andersson. Dynamics and control of electric power systems. In Lecture Notes, ETH Zurich, 2012.

[28] J. Machowski, J. W. Bialek, and J. R. Bumby. Power Systems Dynamics Stability and Control. John Wiley \& Sons, Ltd, second edition, 2008.

[29] P. V. Mieghem. Graph Spectra for Complex Networks. Cambridge University Press, 2011.

[30] B. Gao, G. K. Morison, and P. Kundur. Voltage stability evaluation using modal analysis. IEEE Transactions on Power Systems, 7(4):1529-1542, 1992. 
[31] B. H. Lee and K. Y. Lee. Dynamic and static voltage stability enhancement of power systems. IEEE Transactions on Power Systems, 8(1):231-238, 1993.

[32] L. Gan, N. Li, U. Topcu, and S. H. Low. Optimal power flow in tree networks. In Proc. 52nd IEEE Conference on Decision and Control, pages 2313-2318, 2013.

[33] H. Glavitsch and R. Bacher. Optimal power flow algorithms. In Analysis and Control System Techniques for Electric Power Systems, ETH Zurich, 1991.

[34] R. Madani, S. Sojoudi, and J. Lavaei. Convex relaxation for optimal power flow problem: mesh networks. In Proc. Asilomar Conference on Signals, Systems and Computers, 2013.

[35] S. Boyd and L. Vandenberghe. Convex Optimization. Cambridge University Press, 2004.

[36] H. Farhangi. The path of the smart grid. IEEE Power Energy Magazine, 8(1):18-28, 2010.

[37] R. Srikant. The Mathematics of Internet Congestion Control. Cambridge, MA: Birkhäuser, 2004.

[38] E. Styvaktakis. Automatic classification of power system events using rms voltage measurements. In Proc. Power Engineering Society Summer Meeting, pages 824-829, 2002.

[39] R. Kang and M. McCulloch. Identification of electric power system stress through feeder voltage measurement. In Proc. IEEE PES Innovative Smart Grid Technology Europe, 2014.

[40] A. Papachristodoulou. Scalable analysis of nonlinear systems using convex optimization. PhD dissertation, California Institute of Technology, 2005.

[41] H. K. Khalil. Nonlinear Systems. Prentice Hall, Upper Saddle River, New Jersey, third edition, 2002.

[42] J. K. Hale and S. M. V. Lunel. Introduction to Functional Differential Equations, volume 99. Springer-Verlag New York, Inc, 1993.

\section{Appendix}

Proof of Theorem 1. At the equilibria,

$$
\begin{gathered}
\mathbf{1}_{1 \times m} D_{g} \omega_{g}^{*}+\mathbf{1}_{1 \times n} D_{l} \omega_{l}^{*}=\mathbf{1}_{1 \times m}\left(P_{M}^{*}-P^{\mathcal{G}}\right) \\
-\mathbf{1}_{1 \times n}\left(P_{L}^{*}+P^{\mathcal{L}}\right) \\
\omega_{i}^{*}=\omega_{j}^{*},(i, j) \in \mathcal{E} \\
P_{C}^{*}-P_{M}^{*}=R^{-1} \omega_{g}^{*} \\
\mathbf{1}_{1 \times m} K_{M}^{-1} B\left(P_{M}^{*}-P_{C}^{*}\right)=0
\end{gathered}
$$

hold, which result in $\omega_{g}^{*}=\mathbf{0}_{m \times 1}, P_{L}^{*}=\mathbf{0}_{n \times 1}, \mathbf{1}_{1 \times m}\left(P_{M}^{*}-\right.$ $\left.P^{\mathcal{G}}\right)-\mathbf{1}_{1 \times n} P^{\mathcal{L}}=0, P_{C}^{*}=P_{M}^{*}$ and $A_{0} A_{0}^{T}\left[C P_{C}^{* T}, P_{C}^{\prime * T}\right]^{T}=$ $\mathbf{0}_{(m+n) \times 1}$. Using Lemma 1, the last equation leads to

$$
\left[\begin{array}{c}
C P_{C}^{*} \\
P_{C}^{\prime *}
\end{array}\right]^{T}\left[\begin{array}{c}
\Gamma_{1} \\
\Gamma_{2}
\end{array}\right] A A^{T}\left[\begin{array}{c}
\Gamma_{1} \\
\Gamma_{2}
\end{array}\right]^{T}\left[\begin{array}{c}
C P_{C}^{*} \\
P_{C}^{\prime *}
\end{array}\right]=0 .
$$

Due to $A A^{T} \succ 0[29], \Gamma_{1}^{T} C P_{C}^{*}+\Gamma_{2}^{T} P_{C}^{\prime *}=\mathbf{0}_{(m+n-1) \times 1}$ holds which indicates $c_{i} P_{C_{i}}^{*}=c_{j} P_{C_{j}}^{*}, \forall i, j \in \mathcal{G}$. Thus, $P_{C}^{*}$ is the unique optimal solution of problem (12). According to Remark 3, the equilibrium point of system (15) is unique.
Proof of Theorem 2. Define a candidate Lyapunov function for the overall subsystem as

$$
\begin{aligned}
V_{1}= & \frac{1}{2} \omega_{g}^{T} M \omega_{g}+\frac{1}{2}\left(\alpha-\alpha^{*}\right)^{T} A T A^{T}\left(\alpha-\alpha^{*}\right)+\frac{1}{2}\left(P_{M}\right. \\
& \left.-P_{M}^{*}\right)^{T} T_{T G} R\left(P_{M}-P_{M}^{*}\right)+\frac{1}{2}\left(P_{C}-P_{C}^{*}\right)^{T} B^{-1} R \\
& \times\left(P_{C}-P_{C}^{*}\right)+\frac{1}{2}\left(P_{C}^{\prime}-P_{C}^{\prime *}\right)^{T}\left(P_{C}^{\prime}-P_{C}^{\prime *}\right) .
\end{aligned}
$$

If $K_{M}=\kappa C R^{-1} B$ and $K_{N}=\kappa I_{n}$,

$$
\begin{aligned}
\dot{V}_{1}= & -\omega_{g}^{T} D_{g} \omega_{g}-\omega_{l}^{T} D_{l} \omega_{l}-P_{L}^{T} \omega_{l} \\
& -\left(P_{M}-P_{C}\right)^{T} R\left(P_{M}-P_{C}\right) \\
& -\kappa\left[\begin{array}{c}
C P_{C} \\
P_{C}^{\prime}
\end{array}\right]^{T}\left[\begin{array}{c}
\Gamma_{1} \\
\Gamma_{2}
\end{array}\right] A A^{T}\left[\begin{array}{c}
\Gamma_{1} \\
\Gamma_{2}
\end{array}\right]^{T}\left[\begin{array}{c}
C P_{C} \\
P_{C}^{\prime}
\end{array}\right] \leq 0
\end{aligned}
$$

where $P_{L}^{T} \omega_{l} \geq 0$ is always true. Moreover, $\dot{V}_{1}=0$ only when $\omega_{g}=\mathbf{0}_{m \times 1}, \omega_{l}=\mathbf{0}_{n \times 1}, P_{M}=P_{C}$ and $\left[\Gamma_{1}^{T}, \Gamma_{2}^{T}\right]\left[P_{C}^{T} C, P_{C}^{\prime^{T}}\right]^{T}=\mathbf{0}_{(m+n-1) \times 1}$, which only happens at the equilibrium point. Since $V_{1}$ is radially unbounded, using Krasovskii-LaSalle principle [41], the equilibrium point is asymptotically stable.

Proof of Theorem 3. At the equilibria, Equation (19) holds. Following the proof of Theorem 1, the equilibrium point of system (16) is unique and $P_{C}^{*}$ is the optimal solution of problem (12). To show the asymptotic stability of the equilibrium, define a candidate LyapunovKrasovskii functional as

$$
\begin{aligned}
V_{2}(t)= & \frac{1}{2} \omega_{g}(t)^{T} M \omega_{g}(t)+\frac{1}{2}\left(\alpha(t)-\alpha^{*}\right)^{T} A T A^{T}(\alpha(t) \\
& \left.-\alpha^{*}\right)+\frac{1}{2}\left(P_{M}(t)-P_{M}^{*}\right)^{T} T_{T G} R\left(P_{M}(t)-P_{M}^{*}\right) \\
& +\frac{1}{2}\left(P_{C}(t)-P_{C}^{*}\right)^{T} B^{-1} R\left(P_{C}(t)-P_{C}^{*}\right) \\
& +\frac{1}{2}\left(P_{C}^{\prime}(t)-P_{C}^{* *}\right)^{T}\left(P_{C}^{\prime}(t)-P_{C}^{\prime *}\right) \\
& +\frac{\kappa}{2} \sum_{(i, j) \in \mathcal{E}}\left(\int_{t-\tau_{j i}}^{t}\left(c_{j} P_{C_{j}}(\beta)-c_{j} P_{C_{j}}^{*}\right)^{2} d \beta\right. \\
& \left.+\int_{t-\tau_{i j}}^{t}\left(c_{i} P_{C_{i}}(\beta)-c_{i} P_{C_{i}}^{*}\right)^{2} d \beta\right)
\end{aligned}
$$

where $\kappa>0$. Let $K_{M}=\kappa C R^{-1} B$ and $K_{N}=\kappa I_{n}$. Differentiating $V_{2}$ with respect to time results in

$$
\begin{aligned}
\dot{V}_{2}(t)= & -\omega_{g}(t)^{T} D_{g} \omega_{g}(t)-\omega_{l}(t)^{T} D_{l} \omega_{l}(t)-P_{L}(t)^{T} \omega_{l}(t) \\
& -\left(P_{M}(t)-P_{C}(t)\right)^{T} R\left(P_{M}(t)-P_{C}(t)\right) \\
& -\frac{\kappa}{2} \sum_{(i, j) \in \mathcal{E}}\left(\left(c_{i} P_{C_{i}}(t)-c_{j} P_{C_{j}}\left(t-\tau_{j i}\right)\right)^{2}\right. \\
& \left.+\left(c_{j} P_{C_{j}}(t)-c_{i} P_{C_{i}}\left(t-\tau_{i j}\right)\right)^{2}\right) \leq 0 .
\end{aligned}
$$


Moreover, $\dot{V}_{2}(t)=0$ yields that $\omega_{g}(t)=\mathbf{0}_{m \times 1}, \omega_{l}(t)=$ $\mathbf{0}_{n \times 1}, P_{M}(t)=P_{C}(t), c_{i} P_{C_{i}}(t)=c_{j} P_{C_{j}}\left(t-\tau_{j i}\right), c_{i} P_{C_{i}}(t-$ $\left.\tau_{i j}\right)=c_{j} P_{C_{j}}(t),(i, j) \in \mathcal{E}$ are true. Substituting these equalities into (16) leads to that $P_{M}(t), P_{C}(t)$ are constant, which only happens at the equilibrium point. Since $V_{2}$ is radially unbounded, using LaSalle's invariance principle for time-delay systems [42], the equilibrium point is asymptotically stable.

Proof of Theorem 4. At the equilibria, $n_{T C}^{0} v_{l}^{*}+$ $v_{l}^{0} n_{T C}^{*}=\mathbf{0}_{n \times 1}$ and $Q_{L}^{+*}-Q_{L}^{-*}+Q+L v_{l}^{*}=\mathbf{0}_{n \times 1}$ hold. So a one-to-one correspondence holds between $Q_{L}^{+*}-Q_{L}^{-*}+Q$ and $v_{l}^{*}$ under Assumption 3. Since $Q_{L_{i}}^{+*} Q_{L_{i}}^{-*}=0, i \in \mathcal{L}$ are always true (otherwise, $v_{i}^{*} \geq \bar{v}_{i}$ and $v_{i}^{*} \leq \underline{v}_{i}$ hold which leads to contradiction), the equilibrium point of system (18) is unique. To show the asymptotic stability, consider a candidate Lyapunov function for the overall subsystem as

$$
\begin{aligned}
V_{3}= & \frac{1}{2}\left(v_{l}-v_{l}^{*}\right)^{T} \epsilon v_{l}^{0}\left(v_{l}-v_{l}^{*}\right) \\
& +\frac{1}{2}\left(n_{T C}-n_{T C}^{*}\right)^{T} T_{T} E v_{l}^{0}\left(n_{T C}^{0}\right)^{-1}\left(n_{T C}-n_{T C}^{*}\right) \\
& +\frac{1}{2}\left(Q_{L}^{+}-Q_{L}^{+*}\right)^{T}\left(H^{+}\right)^{-1}\left(Q_{L}^{+}-Q_{L}^{+*}\right) \\
& +\frac{1}{2}\left(Q_{L}^{-}-Q_{L}^{-*}\right)^{T}\left(H^{-}\right)^{-1}\left(Q_{L}^{-}-Q_{L}^{-*}\right) .
\end{aligned}
$$

Differentiating $V_{3}$ with respect to time to get

$$
\begin{aligned}
\dot{V}_{3}= & -\left(n_{T C}^{0} v_{l}+v_{l}^{0} n_{T C}\right)^{T} E\left(n_{T C}^{0}\right)^{-1}\left(n_{T C}^{0} v_{l}+v_{l}^{0} n_{T C}\right) \\
& -\left(v_{l}-v_{l}^{*}\right)^{T}\left(Q_{L}^{+}-Q_{L}^{+*}\right)+\left(v_{l}-v_{l}^{*}\right)^{T}\left(Q_{L}^{-}-Q_{L}^{-*}\right) \\
& -\left(v_{l}-v_{l}^{*}\right)^{T} L\left(v_{l}-v_{l}^{*}\right)+\left(Q_{L}^{+}-Q_{L}^{+*}\right)^{T}\left(v_{l}-\bar{v}\right)_{\mathbf{o}_{n \times 1}} \\
& +\left(Q_{L}^{-}-Q_{L}^{-*}\right)^{T}\left(-v_{l}+\underline{v}\right)_{\mathbf{o}_{n \times 1}}^{-\underline{Q}_{L}} .
\end{aligned}
$$

Note that

$$
\begin{aligned}
& \left(Q_{L_{i}}^{+}-Q_{L_{i}}^{+*}\right)\left(v_{i}-\bar{v}_{i}\right)_{0}^{\bar{Q}_{L_{i}}} \stackrel{(\mathrm{i})}{\leq}\left(Q_{L_{i}}^{+}-Q_{L_{i}}^{+*}\right)\left(v_{i}-\bar{v}_{i}\right) \\
& =\left(Q_{L_{i}}^{+}-Q_{L_{i}}^{+*}\right)\left(v_{i}-v_{i}^{*}\right)+\left(Q_{L_{i}}^{+}-Q_{L_{i}}^{+*}\right)\left(v_{i}^{*}-\bar{v}_{i}\right) \\
& \stackrel{(\mathrm{ii})}{\leq}\left(Q_{L_{i}}^{+}-Q_{L_{i}}^{+*}\right)\left(v_{i}-v_{i}^{*}\right) .
\end{aligned}
$$

Explanation for (i): When $\left(v_{i}-\bar{v}_{i}\right)_{0}^{\bar{Q}_{L_{i}}}$ is inactive (so that it equals to zero), either $Q_{L_{i}}^{+}=0$ and $v_{i}-\bar{v}_{i} \leq 0$ (otherwise, it is positive and then $\left(v_{i}-\bar{v}_{i}\right)_{0}^{\bar{Q}_{L_{i}}}$ would be active which indicates the equality case), or $Q_{L_{i}}^{+}=$ $\bar{Q}_{L_{i}}$ and $v_{i}-\bar{v}_{i} \geq 0$ (otherwise, it is negative and then $\left(v_{i}-\bar{v}_{i}\right)_{0}^{\bar{Q}_{L_{i}}}$ would be active which indicates the equality case) hold. Since $0 \leq Q_{L_{i}}^{+*} \leq \bar{Q}_{L_{i}}$ is always true, for both $\operatorname{cases}\left(Q_{L_{i}}^{+}-Q_{L_{i}}^{+*}\right)\left(v_{i}-\bar{v}_{i}\right)_{0}^{\bar{Q}_{L_{i}}}=0 \leq\left(Q_{L_{i}}^{+}-Q_{L_{i}}^{+*}\right)\left(v_{i}-\bar{v}_{i}\right)$ holds. For the active case, $\left(Q_{L_{i}}^{+}-Q_{L_{i}}^{+*}\right)\left(v_{i}-\bar{v}_{i}\right)_{0}^{\bar{Q}_{L_{i}}}=$ $\left(Q_{L_{i}}^{+}-Q_{L_{i}}^{+*}\right)\left(v_{i}-\bar{v}_{i}\right)$ holds. Explanation for (ii): When $Q_{L_{i}}^{+*}=0, v_{i}^{*} \leq \bar{v}_{i}$ holds. When $Q_{L_{i}}^{+*}=\bar{Q}_{L_{i}}, v_{i}^{*} \geq \bar{v}_{i}$ holds. When $0<Q_{L_{i}}^{+*}<\bar{Q}_{L_{i}}, v_{i}^{*}=\bar{v}_{i}$ holds. So $\left(Q_{L_{i}}^{+}-\right.$ $\left.Q_{L_{i}}^{+*}\right)\left(v_{i}^{*}-\bar{v}_{i}\right) \leq 0$ is true. Similarly, all projections in $\dot{V}_{3}$ can be removed so that

$$
\begin{aligned}
\dot{V}_{3} \leq & -\left(n_{T C}^{0} v_{l}+v_{l}^{0} n_{T C}\right)^{T} E\left(n_{T C}^{0}\right)^{-1}\left(n_{T C}^{0} v_{l}+v_{l}^{0} n_{T C}\right) \\
& -\left(v_{l}-v_{l}^{*}\right)^{T} L\left(v_{l}-v_{l}^{*}\right) \\
& -\left(v_{l}-v_{l}^{*}\right)^{T}\left(Q_{L}^{+}-Q_{L}^{+*}\right)+\left(v_{l}-v_{l}^{*}\right)^{T}\left(Q_{L}^{-}-Q_{L}^{-*}\right) \\
& +\left(Q_{L}^{+}-Q_{L}^{+*}\right)^{T}\left(v_{l}-v_{l}^{*}\right)-\left(Q_{L}^{-}-Q_{L}^{-*}\right)^{T}\left(v_{l}-v_{l}^{*}\right) \\
= & -\left(n_{T C}^{0} v_{l}+v_{l}^{0} n_{T C}\right)^{T} E\left(n_{T C}^{0}\right)^{-1}\left(n_{T C}^{0} v_{l}+v_{l}^{0} n_{T C}\right) \\
& -\left(v_{l}-v_{l}^{*}\right)^{T} L\left(v_{l}-v_{l}^{*}\right) \leq 0
\end{aligned}
$$

where the last inequality is true due to $E \succ 0, n_{T C}^{0} \succ 0$ and $L \succ 0$. If $\dot{V}_{3}=0$ holds, $n_{T C}^{0} v_{l}+v_{l}^{0} n_{T C}=\mathbf{0}_{n \times 1}$, $v_{l}=v_{l}^{*}$ and $Q_{L}^{+}-Q_{L}^{-}+Q+L v_{l}=\mathbf{0}_{n \times 1}$ hold which only happens at the equilibrium point. Since $V_{3}$ is radially unbounded, using Krasovskii-LaSalle principle [41], the equilibrium point is asymptotically stable. 